\title{
On Some Properties of the Quasi-Moment-Method Pathloss Model Calibration
}

\author{
Ayorinde Ayotunde, Adelabu Michael, Muhammed Hisham, Okewole Francis, Mowete Ike* \\ Department of Electrical \& Electronics Engineering, Faculty of Engineering, University of Lagos, Akoka, Lagos, Nigeria \\ Email address: \\ aayorinde@unilag.edu.ng (A. Ayotunde), madelabu@unilag.edu.ng (A. Michael), hmuhammad@unilag.edu.ng (M. Hisham). \\ fokewole@unilag.edu.ng (O. Francis), amowete@unilag.edu.ng (M. Ike) \\ *Corresponding author
}

\section{To cite this article:}

Ayorinde Ayotunde, Adelabu Michael, Muhammed Hisham, Okewole Francis, Mowete Ike. On Some Properties of the Quasi-Moment-Method Pathloss Model Calibration. Journal of Electrical and Electronic Engineering. Vol. 9, No. 4, 2021, pp. 129-146.

doi: $10.11648 /$ j.jeee.20210904.15

Received: July 15, 2021; Accepted: August 2, 2021; Published: August 7, 2021

\begin{abstract}
Certain properties of the recently introduced Quasi-Moment-Method (QMM) for the calibration of basic radiowave propagation pathloss models are systematically examined in this paper. Using measurement data concerning three different routes located in a smart campus environment and made available in the open literature, the paper, in particular, investigates the effects of size of pathloss measurement data on the outcomes of the QMM calibration of nine basic pathloss models: namely, COST 231-urban and sub-urban cities models, ECC33-large and medium sized cities models, and the Egli, Ericsson, Hata, Lee, and SUI- 'Terrain A' models. Computational results reveal that for the data sizes considered, and in the cases of the basic COST 231 and Hata models, which share identical correction factors for receiver antenna height, the 'model calibration matrix' becomes ill-conditioned for one choice of basis functions. The corresponding calibrated models, however, still predict pathloss with accuracy typical of the QMM. For example, Root Mean Square Error (RMSE) outcomes of predictions due to the calibration of these models, emerged as approximately the same for these three models; with values of $6.03 \mathrm{~dB}$ (Route A), $7.96 \mathrm{~dB}$ (Route B), and $6.19 \mathrm{~dB}$ (Route C). The results also show that when model calibration utilizes measurement data for distances further away from the transmitters (by ignoring measurement data for radial distances less than $100 \mathrm{~m}$ away from the transmitters) significant improvements in RMSE metrics were recorded. The paper, in terms of the eigenvalues of the model calibration matrices, further examined the responses of these models to calibration with large-sized measurement data, to find that the model calibration matrices remained characterized, in each case, by a distinctly dominant eigenvalue. An important conclusion arising from the results of the investigations is that whereas the QMM model calibration process may lead, in some cases, and when large-sized measurement data is involved, to 'badly-scaled' model calibration matrices, the calibrated models still record very good assessment metrics. Computational results also reveal that with large-sized data sets, QMM models yield pathloss predictions with excellent (close to $0 \mathrm{~dB}$ ) mean prediction errors.
\end{abstract}

Keywords: Quasi-Moment-Method, Model Calibration Matrix, Eigenvalues, Smart Campus

\section{Introduction}

A few decades ago, Durgin, Rappaport and $\mathrm{Xu}$, [1], predicted that with the then advent in the USA, of the National Information Infrastructure (NII) systems, campus-wide wireless networks will proliferate in universities. They also suggested that because NII systems were designed to use frequencies higher than Personal Communications Systems (PCS) and the cellular systems of that era, pathloss associated with NII signals propagating into buildings and along corridors will require new prediction models. Wireless Local Area Networks (WLANs) have since become routine features in virtually all universities, and the channel modeling issues arising from the introduction of new standards and services continue to engage the attention of the scientific community. In quite a few cases, the focus of research attention is establishing, usually through empirical investigations, that the network of interest will function effectively in the campus environment. Durgin et al [1], for example, utilized extensive field measurements of 
outdoor-to-indoor propagation pathloss concerning a 5.85 $\mathrm{GHz}$ wireless link to characterize channels typical of residential and campus environments. Cheng et al [2], ascertained, through the analysis of pathloss measurement data for a university campus, that a $15 \mathrm{GHz}$ channel represented a suitable choice for the deployment of $5 \mathrm{G}$ networks in the university. Results of empirical investigations carried out by Aborahama et al [3] on a $28 \mathrm{GHz}$ network deployed at the campus of the American University of Sharjar (AUS) in the United Arab Emirates, were utilized for the evaluation of the shadow fading characteristics of $5 \mathrm{G}$ communications in that environment. A similar study was reported by Nwawelu et al [4], whose investigations focused on a $2.4 \mathrm{GHz}$ network deployed at the Nsukka campus of the University of Nigeria. In their contribution, Fraga-Lamas et al [5], utilized measurement results for a campus extending over an area of $26000 \mathrm{~m}^{2}$, to evaluate the performance of a network simulator, implemented for a smart campus scenario. A similar contribution was reported by Han et al [6], who utilized measurement data (as provided by a University of Colorado repository) concerning pathloss for a campus environment for the validation of predictions by a network simulator.

Some of the other investigations of interest to wireless networks deployed in university campuses either compared the performances of existing pathloss models or developed empirical models for the networks. This category includes investigations conducted by Ogunjide et al [7], who took measurements at the Gidan Kwano Campus of the Federal University of Technology Minna, Nigeria. The paper reported outcomes of the performance evaluation of certain basic prediction models as well as empirical model developed with the use of the measurement data. Ramos et al [8] focused on pathloss over short propagation ranges in the $27 \mathrm{GHz}$ to $40 \mathrm{GHz}$ frequency regime, and developed a Fuzzy-based empirical model, with the measurement data from a university campus in Rio de Janerio, Brazil. The Artificial Neural Network (ANN) model developed by Olajide and Samson [9] utilized measurement data from $2.4 \mathrm{GHz}$ networks in two campuses of the Federal University Oye-Ekiti, Nigeria. A corridor propagation case was reported by Femi-Jemilohun and Walker [10], whose empirical model for the $2.4 \mathrm{GHz}$ frequency band is said to be suitable for use with office and campus environments. Unlike the research works briefly described in the foregoing discussions, which focused on pathloss issues associated with outdoor-to-indoor propagation, a number of campus-network studies limited attention only to outdoor, large scale pathloss cases. Many of the investigations, covering different network types and services, were concerned only with the evaluation prediction ability of the existing basic models. Ogbeide and Aikhoje [11], for example, compared predictions by the basic models with field measurement for a cellular (GSM standards) communication network at the Ugbowo campus of the University of Benin, Nigeria. Oyetunji [12], for the FM broadcast station at the Federal University of Technology, Akure, Nigeria, utilized pathloss measurements to evaluate the prediction performance of irregular terrain models. Outcomes of a measurement campaign conducted for the purposes of characterizing outdoor pathloss in 'campus-like' environments were reported by De-Luca et al, [13], who also utilized the results for the evaluation of some models. In a pair of companion papers, Popoola et al $[14,15]$ presented results of the performance evaluation of five pathloss prediction models, using the results of extensive field measurements carried out over three routes at the campus of the Covenant University, Ota, Nigeria. Although like many similar publications, the comparisons reported in [11, 14] suggested that the basic Okumura-Hata and the COST231-Hata models provide the best results for the campus environments investigated, it has since been demonstrated [16-18], that when basic models are calibrated by the recently introduced Quasi-Moment-Method [16], the calibrated ECC-33 models provide the best prediction metrics.

Heretofore, QMM-calibration of the basic pathloss models involved measurement data sizes of less than 100, [17, 18], so that the ability of the model calibration tool to handle large data sets remained unexplored. It is consequently the main objective of this paper, to examine the nature of the characterizing properties of the QMM, when field measurement data size is in excess of 400 . And to that end, field measurement data freely made available by Popoola et al, $[14,15]$ is utilized for the calibration of nine (9) basic pathloss models: COST231 (Urban and Sub-Urban), ECC-33 (Large-and Medium cities), Egli, Ericsson, Okumura-Hata, Lee, and SUI-'Terrain A' models. As may be expected, a number of interesting features were revealed by the computational results. First, the model 'calibration matrix' for the COST231 and Okumura-Hata models, (which share similar correction factors for mobile station antenna height) becomes ill-conditioned when measurement data size exceeds 400. Second, in terms of Root Mean Square prediction Error (RMSE), the solution of the QMM calibration problem virtually converges, when the basic models have similar structures. On the other hand, corresponding Mean Prediction Error (MPE) values differ significantly. Third, the model calibration matrix for each model remains characterized by a single predominant eigenvalue.

The paper in section 2, gives a brief description of the QMM model calibration process, after which the outcomes of the calibration of the nine models using measurement data for three routes (A-496; B-547; C-746) are presented. Using graphical information and tabulated metrics, section 3 evaluates the performances of the calibrated models through comparisons of measurements and predictions, for a number of data size scenarios.

\section{The Quasi-Moment-Method}

The fundamental idea in the Quasi-Moment-Method calibration process is to utilize field measurement data for the calibration of the generic basic pathloss prediction model 
given as

$$
P_{l b}=\varphi_{1}(d)+\varphi_{2}(d)+\ldots+\varphi_{N}(d),
$$

to obtain a corresponding calibrated model defined by

$$
P_{l b c}=\kappa_{1} \varphi_{1}(d)+\kappa_{2} \varphi_{2}(d)+\ldots+\kappa_{N} \varphi_{N}(d)
$$

such that the Euclidean semi-norm of the error function

$$
\varepsilon=\left\|P_{l b}-P_{l b c}\right\|_{2}
$$

assumes its lowest possible value, [16-18]. The calibration coefficients $\kappa_{n}$ appearing in (2) derive directly from the matrix operations defined by

$$
(\mathrm{K})=[\Phi]^{-1}(\mathrm{P})
$$

in which the calibration coefficients are the entries into the column vector $(\mathrm{K})$, and where the $\mathrm{mn}^{\text {th }}$ element of the model calibration matrix is given by, [16],

$$
[\Phi]_{m n}=\left\langle\varphi_{m}, \varphi_{n}\right\rangle=\sum_{k=1}^{L} \varphi_{m}\left(d_{k}\right) \varphi_{n}\left(d_{k}\right) \text {. }
$$

And the $\mathrm{n}^{\text {th }}$ member of the column vector $(\mathrm{P})$ is given by

$$
(P)_{n}=\left\langle\varphi_{n}, p_{\text {mea }}\right\rangle=\sum_{k=1}^{L} \varphi_{n} p_{\text {mea }}\left(d_{k}\right)
$$

provided that it is understood that $p_{\text {mea }}\left(d_{k}\right)$ stands for the pathloss measurement at the distance $d_{k}$ away from the transmitter of interest, and that there are a total of ' $L$ ' such measurements.

\subsection{The QMM-Calibrated Models}

With the use of the basic model calibration process described by (1) to (6) above, nine basic pathloss prediction models were calibrated with measurement data provided by $[14,15]$, for three different routes.

\subsubsection{QMM Models for Route A}

For this route, a total of 496 measurements, covering $69 m \leq d \leq 882 m$ are available from $[14,15]$, for an $1800 \mathrm{MHz}$ network, outcomes of the QMM-calibration of the models are given below, as follows.

$$
P l_{\text {Urbf }}=\left[\begin{array}{l}
0.000101227087050 * 46.3 \\
-0.000020096920747 * 33.9 \log _{10} f \\
+0.000171718943597 * 13.82 \log _{10} h_{t e} \\
-7.472918129858035 *\left[3.2\left(\log _{10} 11.75 h_{r e}\right)^{2}+4.97\right] \\
+0.000731875737185 * 44.9 * \log _{10} d \\
-0.003711162621845 * 6.55 \log _{10} d \log _{10} h_{t e} \\
+0.000529684182110 * 3
\end{array}\right] * 10^{4}
$$

for the COST231 model (large urban city), when the 'basis function' associated with the correction factor for receiver antenna height is as defined by the $4^{\text {th }}$ term of the model. On the other hand, when this factor is utilized as two 'basis functions', with the constant '4.97' being one, and the remaining terms, the other, the QMM calibrated model emerges as

$$
P l_{u r b}=\left(\begin{array}{l}
0.2257 * 46.3-0.0970 * 33.9 \log _{10} f \\
-2.8652 *\left(-13.82 \log _{10} h_{t e}\right. \\
-3.5984 *\left(-3.2 *\left(\log _{10} 11.75 h_{r e}\right)^{2}\right) \\
5.6862 * 4.97+0.3275 *\left(44.9 \log _{10} d\right) \\
-0.5973 *\left(-6.55 \log _{10} d \log _{10} h_{t e}\right) \\
14.0873 * 3.0
\end{array}\right) .
$$

The results of (7 and 8) represent a consequence of ill-conditioning of model calibration matrix earlier alluded to in the introductory remarks, and it is readily verified that pathloss predictions due to these two models are virtually identical. In both equations (and elsewhere in this paper) the numerical figures displayed in magenta colored fonts are the model calibration coefficients obtained with $h_{t e}=18 \mathrm{~m}$ and $h_{r e}=1.5 m$. Other calibrated models for this route include.

$$
. P_{\text {subf }}=\left[\begin{array}{l}
0.000155965842874 * 46.3 \\
-0.000011550116626 * 33.9 \log _{10} f \\
+0.000126503113433 * 13.82 \log _{10} h_{t e} \\
-6.270838608451705 *\left[3.2\left(\log _{10} 11,75 h_{r e}\right)^{2}+4.97\right] \\
+0.000368355366043 * 44.9 * \log _{10} d \\
-0.001726005540827 * 6.55 \log _{10} d \log _{10} h_{t e}
\end{array}\right] * 10^{4}
$$

and

$$
P l_{\text {Sub }}=\left(\begin{array}{l}
0.0682 * 46.3+0.1115 * 33.9 \log _{10} f \\
-1.3410 *\left(-13.82 \log _{10} h_{t e}\right) \\
-3.2274 *\left(-3.2 *\left(\log _{10} 11.75 h_{r e}\right)^{2}\right) \\
16.7188 * 4.97+0.4512 * 44.9 \log _{10} d \\
0.0781 *\left(-6.55 \log _{10} d \log _{10} h_{t e}\right)
\end{array}\right)
$$

in the case of the COST231 (sub-urban city) models, for which comments concerning (7 and 8) also apply. QMM-calibrated models for ECC33 models were obtained as

$$
P l_{M-\text { city }}=\left(\begin{array}{l}
0.776 * 92.4-5.1796 * 20 \log _{10} d \\
+10.3902 * 20 \log _{10} f+(-1.0345) * 20.41 \\
+15.5151 * 9.83 \log _{10} d \\
+13.6189 *\left(7.894+9.56 \log _{10} f\right) \log _{10} f \\
-2.0412 * 13.98 \log _{10}\left(h_{t e} / 200\right) \\
+4.2507 *\left[\log _{10}\left(h_{t e} / 200\right)\right]\left[-5.8\left(\log _{10} d\right)^{2}\right] \\
+2.9035 *\left(-42.57 \log _{10} h_{r e}-0.585\right) \\
-53.4381 *\left(-13.7 \log _{10} f\right)\left(\log _{10} h_{r e}-0.585\right)
\end{array}\right),
$$


for the basic 'medium city' model, and

$$
P l_{L-C i t y}=\left(\begin{array}{l} 
\\
-0.7104 * 92.4-14.0695 * 20 \log _{10} d \\
-13.1934 * 20 \log _{10} f+1.2960 * 20.41 \\
+33.6042 * 9.83 \log _{10} d \\
+15.0113 *\left(7.894+9.56 \log _{10} f\right) \log _{10} f \\
-5.8220 * 13.98 \log _{10}\left(h_{t e} / 200\right) \\
+4.2529 *\left[\log _{10}\left(h_{t e} / 200\right)\right]\left[-5.8\left(\log _{10} d\right)^{2}\right] \\
+(-100.1715) *\left(-0.759 h_{r e}\right)+6.0821 *(1.862)
\end{array}\right) .
$$

for the corresponding 'Large city' model. For the basic Egli model, QMM calibration yielded

$$
P l_{E g l i}=\left(\begin{array}{l}
1.3724 * 76.3+1.3274 * 20 \log _{10}(f) \\
+0.7378 *\left(-20 \log _{10} h_{t e}\right) \\
\left.+19.7382 *\left(-10 \log _{10} h_{r e}\right)\right)+0.4903 * 40 \log _{10} d
\end{array}\right) .
$$

And the calibrated Ericsson model was obtained as

$$
P l_{\text {Eric }}=\left(\begin{array}{l}
1.0653 * 36.2+0.7543 * 30.2 \log _{10} d \\
\left.-0.9427 *\left(-12.0 \log _{10} h_{t e}\right)\right) \\
-25.2360 * 0.1 \log _{10} h_{t e} \log _{10} d \\
-8.5953 *\left(-3.2\left(\log _{10}\left(11.75 h_{r e}\right)\right)^{2}\right) \\
-0.1747 *\left(\log _{10} f\right)\left(44.49-4.78 \log _{10} f\right)
\end{array}\right)
$$

The basic Hata model, which shares a similar 'receiver height correction factor' with the COST231 models, responded to calibration in this case, to yield

$$
P l_{\text {hataf }}=\left[\begin{array}{l}
0.000002335629772 * 69.55 \\
+0.000000030001488 * 26.16 \log _{10} f \\
-0.000020705989039 *\left(-13.82 \log _{10} h_{t e}\right) \\
-1.271044604952447 *\left(-3.2\left(\log _{10} 11.7554 h_{r e}\right)^{2}+4.97\right) \\
-0.000027825381125 * 44.9 \log _{10} d \\
-0.000175805914014 *\left(-6.55 \log _{10} h_{t e} \log _{10} d\right)
\end{array}\right.
$$

and

$$
P l_{\text {hata }}=\left(\begin{array}{l}
0.3912 * 69.55+0.3015 * 26.16 * \log _{10} f \\
+2.9752 *\left(-13.82 \log _{10} h_{t e}\right) \\
-6.1741 *\left(-3.2\left(\log _{10} 11.7554 h_{r e}\right)^{2}\right) \\
+21.3065 * 4.97-6.2744 * 44.9 \log _{10} d \\
-36.6496 *\left(-6.55 \log _{10} h_{t e} \log _{10} d\right)
\end{array}\right)
$$

Model calibration results for the Lee and SUI (Terrain-A) basic models emerged, respectively, as

$$
P l_{L e e}=\left(\begin{array}{l}
0.7163 * 124.0 \\
+0.6430 * 30.5 \log _{10}(d / 1.6) \\
+0.5922 * 30 \log _{10}(f / 900) \\
-15.8892 *(-3.001)
\end{array}\right)
$$

and

$$
P l_{S U I}=\left(\begin{array}{l}
0.4128 * 78.9373+0.4090 * 47.95 \log _{10}(d / 100) \\
+6.1938 * 6 \log _{10}(f / 2000) \\
+0.5846 *\left(-10.8 \log _{10}\left(h_{r e} / 2000\right)\right)+45.0877 * 1.5
\end{array}\right)
$$

\subsubsection{QMM Models for Route B}

Pathloss measurement data for this route extended over $69 m \leq d \leq 883 m$, and a total of 547 measurements were taken, as reported in $[14,15]$. Outcomes of the QMM calibration of the nine basic models of interest to this paper are provided in what follows. Although model calibration matrices for the COST 231 and Hata models also exhibited ill-conditioning of the type described in section 2.1.1, only models associated with the well-conditioned calibration matrices are presented in this section, and henceforth.

The QMM-calibrated COST 231 models were obtained for this route as

$$
P l_{U r b}=\left(\begin{array}{l}
0.6648 * 46.3-0.0559 * 33.9 \log _{10} f \\
+1.1161 *\left(-13.82 \log _{10} h_{t e}\right) \\
-13.8717 *\left(-3.2\left(\log _{10} 11.75 h_{r e}\right)^{2}\right) \\
+17.8954 * 4.97-8.9832 * 44.9 \log _{10} d \\
-51.0905 *-6.55 \log _{10} d \log _{10} h_{t e} \\
-10.4357 * 3.0
\end{array}\right)
$$

for large urban city model, and

$$
\begin{aligned}
& P l_{\text {Sub }} \\
& * 10^{5}
\end{aligned}\left(\begin{array}{l}
0.6632 * 46.3-0.1341 * 33.9 \log _{10} f \\
+0.3911 *\left(-13.82 * \log _{10} h_{t e}\right) \\
-14.1411 *\left(-3.2\left(\log _{10} 11.75 h_{r e}\right)^{2}\right) \\
+10.5465 * 4.97-6.6999 *\left(44.9 \log _{10} d\right) \\
-38.6212 *\left(-6.55 \log _{10} d \log _{10} h_{t e}\right)
\end{array}\right)
$$

in the case of the sub-urban cities model. Calibration results for the ECC 33 models emerged as

$$
P l_{L-\text { city }}=\left(\begin{array}{l}
-0.7562 * 92.4-15.5395 * 20 \log _{10} d \\
-9.6229 * 20 \log _{10} f+1.1130 * 20.41 \\
+36.1512 * 9.83 \log _{10} d \\
+19.3550 * \log _{10} f\left(7.894+9.56 \log _{10} f\right) \\
+5.3070 *\left(-13.98 \log _{10}\left(h_{t e} / 200\right)\right) \\
+4.0527 \log _{10}\left(h_{t e} / 200\right)\left(-5.8\left(\log _{10}(d)\right)^{2}\right. \\
-83.6627 *\left(-0.759 h_{r e}\right)+4.7108 *(1.862)
\end{array}\right)
$$

for the large cities model, and in the case of medium sized cities, as 


$$
P l_{M-c i t y}=\left(\begin{array}{l}
0.0773 * 92.4-7.1816 *\left(20 \log _{10} d\right) \\
+3.5635 * 20 \log _{10} f \\
-0.7472 * 20.41+19.1452 * 9.83 \log _{10} d \\
+31.2228 *\left(\log _{10} f\right)\left(7.894+9.56 \log _{10} f\right) \\
+3.5820 *\left(-13.98 \log _{10}\left(h_{t e} / 200\right)\right) \\
+4.0514 * \log _{10}\left(h_{t e} / 200\right) *\left(-5.8\left(\log _{10} d\right)^{2}\right) \\
+4.9231 *\left(-42.57 *\left(\log _{10} h_{r e}-0.585\right)\right. \\
-65.8272 *\left(-13.7 \log _{10} f\right)\left(\log _{10} h_{r e}-0.585\right)
\end{array}\right)
$$

The basic Egli and Ericsson models calibrated with measurement data for this route are

$$
P l_{\text {Egli }}=\left(\begin{array}{l}
-3.7712 * 76.3-0.3956 * 20 \log _{10} f \\
-8.0376 *\left(-20 \log _{10} h_{t e}\right) \\
-138.2958 *\left(-10 \log _{10} h_{r e}\right) \\
0.4180 * 40 \log _{10} d
\end{array}\right)
$$

and

$$
P l_{\text {Eric }}=\left(\begin{array}{l}
1.1734 * 36.2+1.1717 * 30.2 \log _{10} d \\
+1.3549 *\left(-12.0 \log _{10} h_{t e}\right) \\
-148.6941 * 0.1 \log _{10} h_{t e} \log _{10} d \\
-10.4651 *\left(-3.2\left(\log _{10} 11.75 h_{r e}\right)^{2}\right) \\
+0.0805 *\left(\log _{10} f\right)\left(44.49-4.78 \log _{10} f\right)
\end{array}\right),
$$

respectively. Following calibration with 'Route B' field measurement data, the basic Hata, Lee and SUI models emerged, modified as described by

$$
P l_{\text {Hata }}=\left(\begin{array}{l}
-1.0631 * 69.55+0.2720 * 26.16 \log _{10} f \\
-18.6491 *\left(-13.82 \log _{10} h_{t e}\right) \\
+15.9805 *\left(-3.2\left(\log _{10} 11.7554 h_{r e}\right)^{2}\right) \\
-12.3744 * 4.97+12.7351 * 44.9 \log _{10} d \\
+67.5120 *\left(-6.55 * \log _{10} h_{t e} \log _{10} d\right.
\end{array}\right)
$$

for the Hata model,

$$
P l_{\text {Lee }}=\left(\begin{array}{l}
0.8825 * 124.0 \\
+0.5482 * 30.5 \log _{10}(d / 1.6) \\
+2.6827 * 30 \log _{10}(f / 900) \\
-0.5218 *(-3.001)
\end{array}\right)
$$

and

$$
P l_{S u i}=\left(\begin{array}{l}
0.4621 * 78.9373 \\
+0.3487 * 47.95 \log _{10}(d / 100) \\
-97.9723 * 6.0 \log _{10}(f / 2000) \\
+0.8337 *\left(-10.8 \log _{10}\left(h_{r e} / 2000\right)\right) \\
+15.7223 * 1.5
\end{array}\right)
$$

in the case of the SUI model, for this route.

\subsubsection{QMM Models for Route C}

A total of 746 measurements extending over the $1 m \leq d \leq 1132 m$ were recorded for this route [14, 15]. And outcomes of the QMM calibration of the basic models with this set of measurement data are as described in what follows, starting with the basic COST231 large urban cities model, for which

$$
P l_{U r b}=\left(\begin{array}{l}
0.9930 * 46.3+0.1426 * 33.9 \log _{10} f \\
+2.0175 *\left(-13.82 \log _{10} h_{t e}\right) \\
-18.9776 *\left(-3.2 *\left(\log _{10} 11.75 h_{r e}\right)^{2}\right)+19.9728 * 4.97 \\
+5.9159 *\left(44.9 \log _{10} d\right) \\
+31.4740 *\left(-6.55 \log _{10} d \log _{10} h_{t e}\right)-28.5822 * 3.0
\end{array}\right)
$$

The QMM-calibrated COST231 (sub-urban cities) model was obtained as

$$
P l_{\text {Sub }}=\left(\begin{array}{l}
1.3573 * 46.3-0.0151 * 33.9 \log _{10} f \\
+1.0613 *\left(-13.82 \log _{10} h_{t e}\right) \\
-15.1288 *\left(-3.2 *\left(\log _{10} 11.75 h_{r e}\right)^{2}\right) \\
+3.3399 * 4.97 \\
-0.0653 *\left(44.9 * \log _{10} d\right) \\
-1.1894 *\left(-6.55 \log _{10} d \log _{10} h_{t e}\right)
\end{array}\right)
$$

whilst those for the ECC 33 models emerged as

$$
P l_{L-\text { city }}=\left(\begin{array}{l}
0.1045 * 92.4-1.0141 * 20.0 \log _{10} d \\
-2.1347 * 20.0 \log _{10} f \\
+0.9998 * 20.41+1.7323 * 9.83 \log _{10} d \\
+0.0508 * \log _{10} f\left(7.894+9.56 \log _{10} f\right) \\
+3.3043 *\left(-13.98 \log _{10}\left(h_{t e} / 200\right)\right) \\
-0.9936 *\left(\log _{10}\left(h_{t e} / 200\right)\right)\left(-5.8\left(\log _{10} d\right)^{2}\right) \\
-30.0573 *\left(-0.759 h_{r e}\right)+16.5165 *(1.862)
\end{array}\right),
$$

in the case of the basic large cities model, and

$$
P l_{M-\text { city }}=\left(\begin{array}{l}
0.4853 * 92.4-2.5272 * 20.0 \log _{10} d \\
-0.8566 * 20.0 \log _{10} f \\
-0.5493 * 20.41+4.7585 * 9.83 \log _{10} d \\
+4.7585 * \log _{10} f\left(7.894+9.56 \log _{10} f\right) \\
+0.8804 *\left(-13.98 * \log _{10} h_{t e} / 200\right) \\
-0.8638 *\left(\log _{10}\left(h_{t e} / 200\right)\right)\left(-5.8\left(\log _{10} d\right)^{2}\right) \\
+2.2822 *\left(-42.57\left(\log _{10} h_{r e}-0.585\right)\right) \\
+24.9060 *\left(-13.7 \log _{10} f\left(\log _{10} h_{r e}-0.585\right)\right)
\end{array}\right),
$$

for the small-medium sized cities model. For the other five basic models, outcomes of QMM calibration emerged as

$$
P l_{E g l i}=\left(\begin{array}{l}
0.3149 * 76.3+0.9024 * 20 \log _{10} f \\
+0.9350 *\left(-20 \log _{10} h_{t e}\right)-42.0734\left(-10 \log _{10} h_{r e}\right) \\
+0.1711 * 40 \log _{10} d
\end{array}\right),
$$

for the Egli model, 


$$
{ }_{\text {Eric }}=\left(\begin{array}{l}
0.3825 * 36.2+0.9610 * 30.2 \log _{10} d \\
+0.1660 *\left(-12.0 \log _{10} h_{t e}\right) \\
-176.6448 * 0.1 \log _{10} h_{t e} \log _{10} d \\
-16.4746 *\left(-3.2\left(\log _{10}\left(11.75 * h_{r e}\right)^{2}\right)\right) \\
0.2207 * \log _{10} f\left(44.49-4.78 \log _{10} f\right)
\end{array}\right),
$$

in the case of the Ericsson model,

$$
P l_{\text {Hata }}=\left(\begin{array}{l}
0.9129 * 69.55+0.5760 * 26.16 \log _{10} f \\
+1.0886 *\left(-13.82 \log _{10} h_{t e}\right) \\
+2.1915 *\left(-3.2\left(\log _{10} 11.7554 h_{r e}\right)^{2}\right) \\
+10.4166 * 4.97-14.7561 * 44.9 \log _{10} d \\
-81.4144 *\left(-6.55 \log _{10} h_{t e} \log _{10} d\right)
\end{array}\right)
$$

for the Hata model, and

$$
P l_{\text {Lee }}=\left(\begin{array}{l}
0.5876 * 124.0+0.2244 * 30.5 \log _{10}(d / 1.6) \\
+4.5201 * 30 \log _{10}(f / 900)-7.4152 *(-3.001)
\end{array}\right)
$$

and

$$
P l_{\text {Sui }}=\left(\begin{array}{l}
1.1227 * 78.9373+0.0300 * 47.95 \log _{10}(d / 100) \\
153.6073 * 6.0 \log _{10}(f / 2000) \\
1.6206 *\left(-10.8 \log _{10}\left(h_{r e} / 2000\right)+20.2447 * 1.5\right.
\end{array}\right)
$$

for the Lee and SUI models, respectively.

\subsection{Alternative Calibration Models}

In order to examine the effects of measurements taken close to the transmitting antenna location, on the performances of the QMM calibrated models, alternative models were developed, using measurement data with starting points significantly farther away from the transmitter, than was the case for the each of the models in section 2.1.

\subsubsection{Route A}

For this route, model calibration was carried out using measurement data extending over $101 m \leq d \leq 882 \mathrm{~m}$, a total of 465 pathloss measurements. Outcomes of the calibrations include

$$
P l_{U r b}=\left(\begin{array}{l}
0.4164 * 46.3-0.0564 * 33.9 \log _{10} f \\
-4.6064 *\left(-13.82 \log _{10} h_{t e}\right) \\
-0.9871 *\left(-3.2\left(\log _{10} 11.75 h_{r e}\right)^{2}\right)-1.8667 * 4.97 \\
+11.7856 *\left(44.9 \log _{10} d\right)+16.8061 * 3.0 \\
+61.5045 *\left(-6.55 \log _{10} d \log _{10} h_{t e}\right)
\end{array}\right)
$$

and

$$
P l_{S u b}=\left(\begin{array}{l}
0.0456 * 46.3+0.2543 * 33.9 \log _{10} f \\
-2.1806 *\left(-13.82 \log _{10} h_{t e}\right) \\
-1.3083 *\left(-3.2\left(\log _{10} 11.75 h_{r e}\right)^{2}\right) \\
+12.9806 * 4.97+10.2455 * 44.9 \log d \\
+53.0941 *\left(-6.55 * \log _{10} d \log _{10} h_{t e}\right)
\end{array}\right)
$$

for the COST231 models, as well as

$$
P l_{L-\text { city }}=\left(\begin{array}{l}
0.7756 * 92.4+25.5614 * 20.0 \log _{10} d \\
+7.3109 * 20.0 \log _{10} f+1.7006 * 20.41 \\
-45.5793 * 9.83 \log _{10} d \\
-12.8898 * \log _{10} f\left(7.894+9.56 \log _{10} f\right) \\
-0.4867 *\left(-13.98 \log _{10}\left(h_{t e} / 200\right)\right) \\
+6.9246 *\left(\log _{10}\left(h_{t e} / 200\right)\right)\left(-5.8\left(\log _{10} d\right)^{2}\right) \\
-3.7692 *\left(-0.759 h_{r e}\right)+20.8918 *(1.862)
\end{array}\right),
$$

and

$$
P l_{M-\text { city }}=\left(\begin{array}{l}
0.9517 * 92.4+38.1837 * 20.0 * \log _{10} d \\
+15.4824 * 20.0 \log _{10} f \\
-0.6267 * 20.41-71.2617 *\left(9.83 \log _{10} d\right) \\
+6.9086 * \log _{10} f\left(7.894+9.56 \log _{10} f\right) \\
+1.7269 *\left(-13.98 \log _{10}\left(h_{t e} / 200\right)\right) \\
+6.9228 *\left(\log _{10}\left(h_{t e} / 200\right)\right)\left(-5.8\left(\log _{10} d\right)^{2}\right) \\
+0.1425 *\left(-42.57\left(\log _{10} h_{r e}-0.585\right)\right. \\
-38.0268 *\left(-13.7 \log _{10} f\left(\log _{10} h_{r e}-0.585\right)\right.
\end{array}\right),
$$

for the ECC33 models. Others are the Egli model, for which

$$
P l_{E g l i}=\left(\begin{array}{l}
0.7094 * 76.3+1.1145 * 20 \log _{10} f \\
-0.2429 *\left(-20 \log _{10} h_{t e}\right)-3.5364 *\left(-10 \log _{10} h_{r e}\right) \\
0.5870 * 40 \log _{10} d
\end{array}\right),
$$

the Ericsson model, whose calibrated version emerged as

$$
P l_{\text {Eric }}=\left(\begin{array}{l}
1.0679 * 36.2+0.6520 * 30.2 \log _{10} \\
-1.8939 *\left(-12.0 \log _{10} h_{t e}\right) \\
+30.1626 * 0.1 \log _{10} h_{t e} \log _{10} d \\
-7.4503 *\left(-3.2\left(\log _{10} 11.75 h_{r e}\right)^{2}\right) \\
-0.3783 * \log _{10} f\left(44.49-4.78 \log _{10} f\right)
\end{array}\right),
$$

the Hata model, with

$$
P l_{\text {Hata }}=\left(\begin{array}{l}
-0.9714 * 69.55+2.2093 * 26.16 \log _{10} f \\
-28.9296 *\left(-13.82 \log _{10} h_{t e}\right) \\
+45.7145 *\left(-3.2\left(\log _{10} 11.7554 h_{r e}\right)^{2}\right) \\
-51.5493 * 4.97+30.1968 * 44.9 \log _{10} d \\
+162.0469 *\left(-6.55 \log _{10} h_{t e} \log _{10} d\right)
\end{array}\right),
$$


as its calibrated version, and the Lee and SUI models, with calibrated outcomes obtained as

$$
P l_{\text {Lee }}=\left(\begin{array}{l}
0.4616 * 124.0+0.7697 * 30.5 \log _{10}(d / 1.6) \\
+1.6141 * 30 \log _{10}(f / 900)-23.9886 *(-3.001)
\end{array}\right)
$$

and

$$
P l_{\text {Sui }}=\left(\begin{array}{l}
0.3599 * 78.9373+0.4896 * 47.95 \log _{10}(d / 100) \\
-28.9879 * 6 \log _{10}(f / 2000) \\
+0.4907 *\left(-10.8 \log _{10}\left(h_{r e} / 2000\right)\right)+41.7352 * 1.5
\end{array}\right),
$$

respectively.

\subsubsection{Route B}

The alternative calibration models for this route derived from a total of 447 pathloss measurements extending between $308 m \leq d \leq 882 m$. And the outcomes of the QMM-calibrations were recorded for the COST231 (urban and suburban) models as

$$
P l_{U r b}=\left(\begin{array}{l}
0.6648 * 46.3-0.0559 * 33.9 \log _{10} f \\
+1.1161 *\left(-13.82 \log _{10} h_{t e}\right) \\
-13.8717 *\left(-3.2\left(\log _{10}\left(11.75 h_{r e}\right)^{2}\right)\right. \\
+17.8954 * 4.97-8.9832 *\left(44.9 \log _{10} d\right) \\
-51.0905 *\left(-6.55 * \log _{10} d \log _{10} h_{t e}\right) \\
-10.4357 * 3.0
\end{array}\right)
$$

and

$$
P l_{S u b}=\left(\begin{array}{l}
0.6632 * 46.3-0.1341 * 33.9 \log _{10} f \\
+0.3911 *\left(-13.82 \log _{10} h_{t e}\right) \\
-14.1411 *\left(-3.2\left(\log _{10}\left(11.75 h_{r e}\right)^{2}\right)\right. \\
+10.5465 * 4.97 \\
-6.6999 *\left(44.9 \log _{10} d\right) \\
-38.6212 *\left(-6.55 \log _{10} d \log _{10} h_{t e}\right)
\end{array}\right) .
$$

The responses of the basic ECC33 models to calibration were in this case, obtained as

$$
P l_{L-\text { city }}=\left(\begin{array}{l}
-0.7562 * 92.4-15.5395 * 20.0 \log _{10} d \\
-9.6229 * 20.0 \log _{10} f \\
+1.1130 * 20.41+36.1512 * 9.83 * \log _{10} d \\
+19.3550 * \log _{10} f\left(7.894+9.56 \log _{10} f\right) \\
+5.3070 *\left(-13.98 \log _{10}\left(h_{t e} / 200\right)\right) \\
+4.0527 * \log _{10}\left(h_{t e} / 200\right)\left(-5.8\left(\log _{10} d\right)^{2}\right) \\
-83.6627 *\left(-0.759 h_{r e}\right)+4.7108 *(1.862)
\end{array}\right),
$$

for the large-city model, and

$$
P l_{M-\text { city }}=\left(\begin{array}{l}
0.0773 * 92.4-7.1816 * 20.0 \log _{10} d \\
+3.5635 * 20.0 \log _{10} f \\
-0.7472 * 20.41+19.1452 * 9.83 \log _{10} d \\
+31.2228 * \log 10(f)\left(7.894+9.56 * \log _{10} f\right) \\
+3.5820 *\left(-13.98 \log 10\left(h_{t e} / 200\right)\right) \\
+4.0514 * \log _{10}\left(h_{t e} / 200\right)\left(-5.8\left(\log _{10} d\right)^{2}\right) \\
+4.9231 *\left(-42.57\left(\log _{10} h_{r e}-0.585\right)\right. \\
-65.8272 *\left(-13.7 \log _{10} f\left(\log _{10} h_{r e}-0.585\right)\right.
\end{array}\right)
$$

in the case of the 'small-medium' sized cities. QMM-calibrations of the basic Egli and Ericsson models for this route yielded

$$
P l_{E g l i}=\left(\begin{array}{l}
-3.7712 * 76.3-0.3956 * 20 \log _{10} f \\
-8.0376 *\left(-20 \log _{10} h_{t e}\right)-138.2958 *\left(-10 \log _{10} h_{r e}\right) \\
+0.4180 * 40 \log _{10} d
\end{array}\right)
$$

and

$$
P l_{\text {Eric }}=\left(\begin{array}{l}
1.1734 * 36.2+1.1717 * 30.2 \log _{10} d \\
+1.3549 *\left(-12.0 * \log _{10} h_{t e}\right) \\
-148.6941 * 0.1 \log _{10} h_{t e} \log _{10} d \\
-10.4651 *\left(-3.2\left(\log _{10} 11.75 h_{r e}\right)^{2}\right) \\
+0.0805 * \log _{10} f^{*}\left(44.49-4.78 \log _{10} f\right)
\end{array}\right)
$$

respectively, whilst the respective calibrated Hata and Lee models emerged as

$$
P l_{\text {Hata }}=\left(\begin{array}{l}
-1.0631 * 69.55+0.2720 * 26.16 \log _{10} f \\
-18.6491 *\left(-13.82 \log _{10} h_{t e}\right) \\
+15.9805 *\left(-3.2\left(\log _{10}\left(11.7554 h_{r e}\right)^{2}\right)\right. \\
-12.3744 * 4.97+12.7351 * 44.9 \log _{10} d \\
+67.5120 *\left(-6.55 \log _{10} h_{t e} \log _{10} d\right)
\end{array}\right)
$$

and

$$
P l_{\text {Lee }}=\left(\begin{array}{l}
0.8825 * 124.0+0.5482 * 30.5 \log _{10}(d / 1.6) \\
+2.6827 * 30 * \log _{10}(f / 900)-0.5218 *(-3.001)
\end{array}\right)
$$

Finally, the basic SUI model's response to the calibration emerged as

$$
P l_{\text {Sui }}=\left(\begin{array}{l}
0.4621 * 78.9373+0.3487 * 47.95 * \log _{10}(d / 100) \\
-97.9723 * 6.0 \log _{10}(f / 2000) \\
+0.8337 *\left(-10.8 * \log _{10}\left(h_{r e} / 2000\right)\right)+15.7223 * 1.5
\end{array}\right) .
$$

\subsubsection{Route C}

Alternative calibration models developed for this route utilized 647 pathloss field measurements covering the radial distance range defined by $100 m \leq d \leq 1132 m$. Basic model calibration results obtained for the COST231 models are given below as 


$$
P l_{U r b}=\left(\begin{array}{l}
0.2397 * 46.3+0.2767 * 33.9 \log _{10} f \\
+1.6029 *\left(-13.82 \log _{10} h_{t e}\right) \\
-18.6878 *\left(-3.2\left(\log _{10} 11.75 h_{r e}\right)^{2}\right) \\
-1.4282 * 4.97+13.6959 *\left(44.9 \log _{10} d\right) \\
+74.7648 *\left(-6.55 * \log _{10} d \log _{10} h_{t e}\right) \\
+11.0247 * 3.0
\end{array}\right)
$$

and

$$
P l_{S u b}=\left(\begin{array}{l}
0.0709 * 46.3+0.3735 * 33.9 \log _{10} f \\
+1.5154 *\left(-13.82 \log _{10} h_{t e}\right) \\
-16.3797 *\left(-3.2\left(\log _{10}\left(11.75 h_{r e}\right)^{2}\right)\right) \\
+6.6529 * 4.97+16.7724 * 44.9 \log _{10} d \\
+91.5651 *\left(-6.55 \log _{10} d \log _{10} h_{t e}\right)
\end{array}\right),
$$

respectively, for the urban and sub-urban cities' models. In the cases of the two ECC33 models, the corresponding models are

$$
P l_{M-\text { city }}=\left(\begin{array}{l}
0.5776 * 92.4+7.9049 * 20.0 \log _{10} d \\
+1.0889 * 20.0 \log _{10} f \\
-0.5191 * 20.4-16.1574 * 9.83 \log _{10} d \\
+0.2796 * \log _{10} f\left(7.894+9.56 \log _{10} f\right) \\
+1.0761 *\left(-13.98 \log _{10}\left(h_{t e} / 200\right)\right) \\
-0.1975 *\left(\log _{10}\left(h_{t e} / 200\right)\left(-5.8\left(\log _{10} d\right)^{2}\right)\right) \\
+1.1501 *\left(-42.57\left(\log _{10} h_{r e}-0.585\right)\right. \\
+33.3913 *\left(-13.7 \log _{10} f\left(\log _{10} h_{r e}-0.585\right)\right)
\end{array}\right)
$$

for the 'small-medium' sized cities model, and

$$
P l_{L-\text { city }}=\left(\begin{array}{l}
-0.2198 * 92.4+25.8928 * 20.0 \log _{10} d \\
-3.5881 * 20.0 \log _{10} f+1.2356 * 20.41 \\
-52.7549 * 9.83 \log _{10} d \\
+1.6062 * \log _{10} f\left(7.894+9.56 \log _{10} f\right) \\
+3.1616 *\left(-13.98 \log _{10}\left(h_{t e} / 200\right)\right) \\
-0.1966 * \log _{10}\left(h_{t e} / 200\right)\left(-5.8\left(\log _{10} d\right)^{2}\right) \\
-52.6714 *\left(-0.759 h_{r e}\right)+19.0976 * 1.862
\end{array}\right)
$$

for the large cities model. The calibrated Egli model was obtained as

$$
P l_{E g l i}=\left(\begin{array}{l}
1.1557 * 76.3+1.7250 * 20 \log _{10} f \\
+2.8814 *\left(-20 \log _{10} h_{t e}\right) \\
-2.5671 *\left(-10 \log _{10} h_{r e}\right) \\
+0.0057 * 40 \log _{10} d
\end{array}\right)
$$

and for the Ericsson model, as

$$
P l_{\text {Eric }}=\left(\begin{array}{l}
2.0279 * 36.2+0.9399 * 30.2 \log _{10} d \\
+1.3523 *\left(-12.0 \log _{10} h_{t e}\right) \\
-224.2710 * 0.1 \log _{10} h_{t e} \log _{10} d \\
-15.5071 *\left(-3.2\left(\log _{10}\left(11.75 h_{r e}\right)^{2}\right)\right) \\
+0.0199 * \log _{10} f\left(44.49-4.78 * \log _{10} f\right)
\end{array}\right)
$$

In response to the QMM calibration with this route's field measurements as earlier described, the Hata model yielded

$$
P l_{\text {Hata }}=\left(\begin{array}{l}
-0.5669 * 69.55+0.0760 * 26.16 \log _{10} f \\
-11.2477 *\left(-13.82 * \log _{10} h_{t e}\right) \\
+1.3876 *\left(-3.2\left(\log _{10}\left(11.7554 h_{r e}\right)^{2}\right)\right) \\
-4.5477 * 4.97-5.9894 * 44.9 \log _{10} d \\
-32.7355 *\left(-6.55 * \log _{10} h_{t e} \log _{10} d\right)
\end{array}\right)
$$

as the calibrated version. And the responses of the Lee and SUI models to QMM calibration, emerged, respectively, for this route, as

$$
P l_{\text {Lee }}=\left(\begin{array}{l}
0.8891 * 124.0 \\
+0.0075 * 30.5 \log _{10}(d / 1.6) \\
+2.6303 * 30 \log _{10}(f / 900) \\
+0.4310 *(-3.001)
\end{array}\right)
$$

and

$$
P l_{S u i}=\left(\begin{array}{l}
1.1227 * 78.9373 \\
+0.0300 * 47.95 \log _{10}(\mathrm{~d} / 100) \\
+153.6073 * 6.0 \log _{10}(\mathrm{f} / 2000) \\
+1.6206 *\left(-10.8 \log _{10}\left(\mathrm{~h}_{\mathrm{re}} / 2000\right)\right) \\
+20.2447 * 1.5
\end{array}\right)
$$

\section{Discussion of Results}

\subsection{Prediction Performances of Route A QMM Models}

With the use of the models presented in $\S 2$, the prediction performances of the calibrated models are evaluated in this section, through comparisons with measurement data.

Figures 1 and 2 compare pathloss predicted by the models of (7) to (18), with pathloss measurements.

Table 1 describes the performances of the models in terms of Root Mean Square Error (RMSE) and Mean Prediction Error (MPE). And it is readily observed from the table that in all cases, the calibrated models perform significantly better than the basic models from which they derive. An interesting feature of the metrics is that the RMSE values generally fall into two broad groups: $5.579 \mathrm{~dB}$ for both ECC 33 models, and $6.038 \mathrm{~dB}$ for the other models: with the SUI-Terrain model being the exception to this observation. Mean Prediction Error (MPE) generally ranged between $0.002 \mathrm{~dB}$ and $0.004 \mathrm{~dB}$, with the Egli model, which recorded an MPE of 0.0006dB and the SUI model $(-1.4206 \mathrm{~dB})$ being exceptions. As a matter of fact, although not shown in the table, Mean Absolute Prediction Error (MAPE) followed the same pattern as the RMSE, in that the ECC33 models recorded $4.0234 \mathrm{~dB}$, whilst the other models (with the exception of the $4.6479 \mathrm{~dB}$ recorded by the SUI model) had $4.6034 \mathrm{~dB}$ as their MAPE values. 

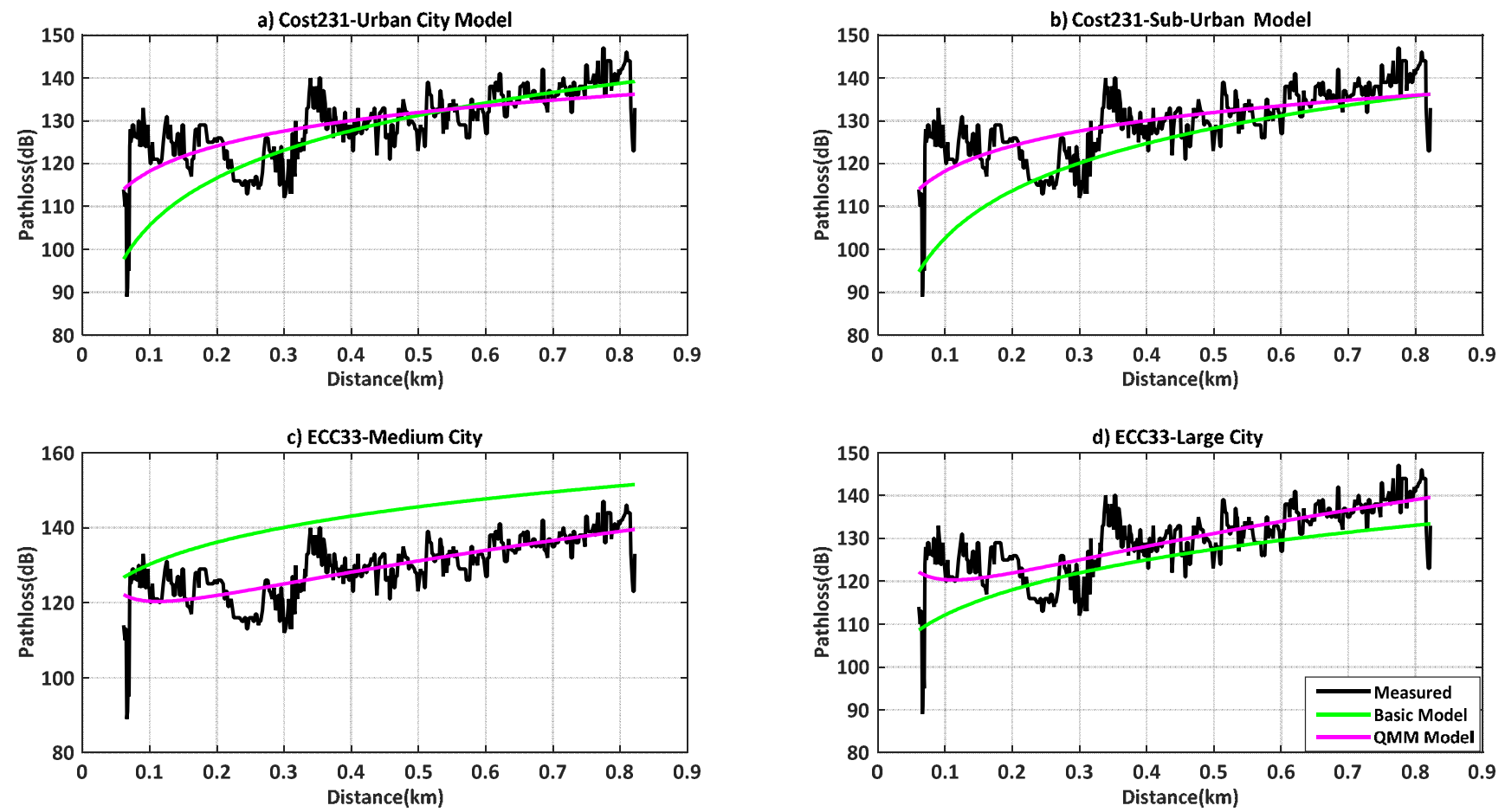

Figure 1. Comparison of predicted and measured pathloss for Route A QMM COST231 and ECC33 Models.
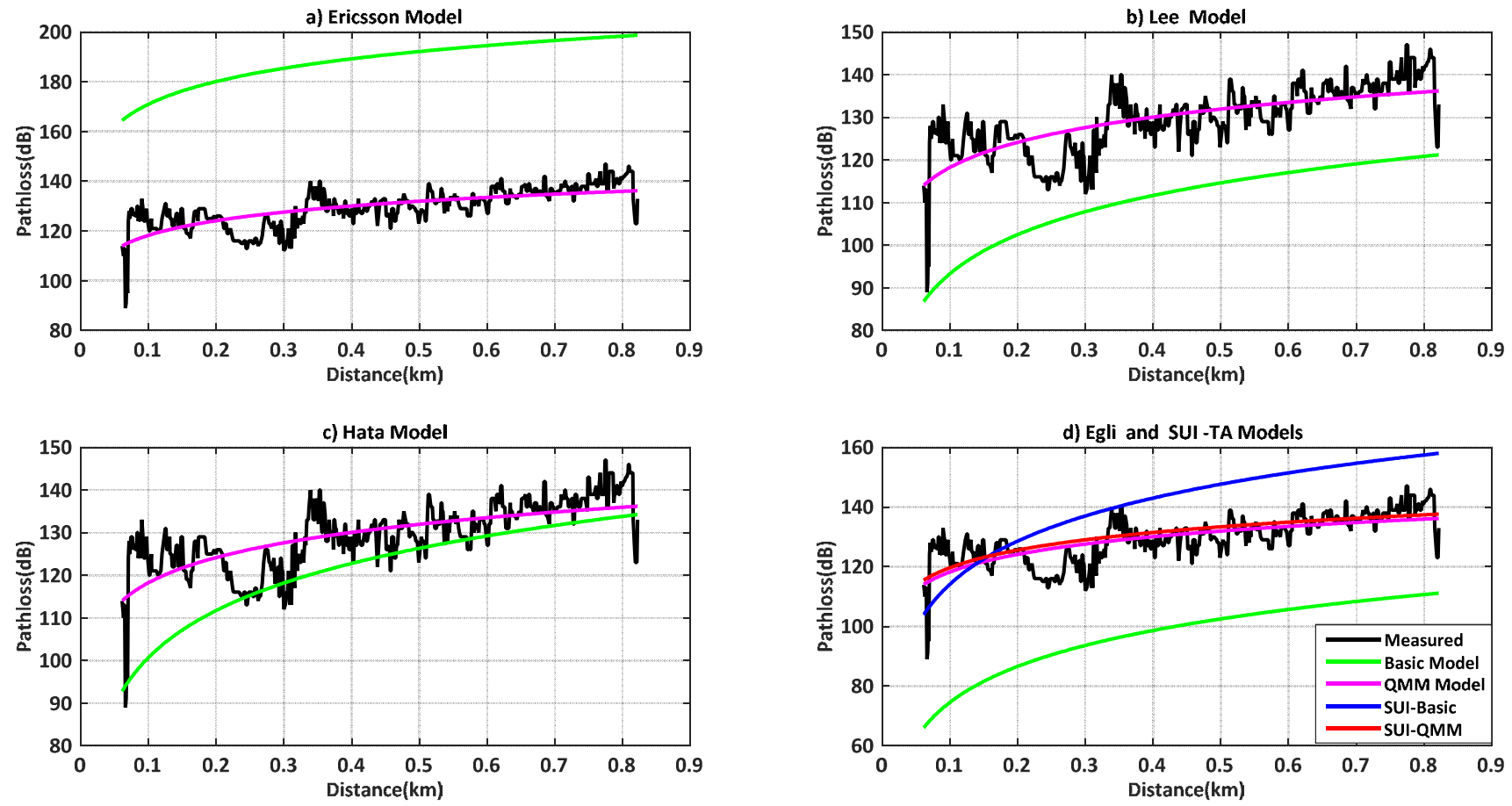

Figure 2. Comparison of predicted and measured pathloss for Route A QMM Egli, Ericsson, Hata, Lee and SUI Models.

Table 1. RMSE and MPE Metrics for Route A Models.

\begin{tabular}{lllll}
\hline \multirow{2}{*}{ Model/Metric } & RMSE (dB) & & MPE (dB) & QMM \\
\cline { 2 - 5 } & Basic & QMM & Basic & 0.0013 \\
\hline COST231-SU & 9.7885 & 6.0383 & 5.9070 & 0.0023 \\
COST231-UR & 8.3290 & 6.0383 & 2.9070 & -0.0018 \\
ECC33-L & 7.5838 & 5.5791 & 4.7266 & -0.0031 \\
ECC33-M & 14.642 & 5.5791 & -13.3872 & -0.0006 \\
RGLI & 33.200 & 6.0383 & 32.1073 & \\
\hline
\end{tabular}




\begin{tabular}{lllll}
\hline \multirow{2}{*}{ Model/Metric } & RMSE (dB) & & MPE (dB) & QMM \\
\cline { 2 - 5 } & Basic & QMM & Basic & -0.0042 \\
ERICSSON & 59.236 & 6.0383 & -58.8442 & 0.0028 \\
HATA & 11.072 & 6.0383 & 7.8528 & 0.0045 \\
LEE & 19.945 & 6.0383 & 18.7454 & -1.4206 \\
SUI & 15.758 & 6.2032 & -12.0162 & \\
\hline
\end{tabular}

The profiles of Figure 3 compare pathloss predicted by the alternative QMM models defined by (37) - (45) with corresponding field measurement data. It is apparent from the profiles in the figure that predictions by all the models (with the exception of the ECC33 models) are virtually coincident.

In addition to supporting this observation, the metrics of Table 2, which share the features earlier described for Table 1, reveal that RMSE improves by about $15 \%$ for the ECC 33 models, and about $12 \%$ for the other models, when the alternative calibration is utilized for this route.

Table 2. RMSE and MPE Metrics for the Alternative Route A Models.

\begin{tabular}{lllll}
\hline \multirow{2}{*}{ Model/Metric } & \multicolumn{3}{c}{ RMSE (dB) } & \multicolumn{3}{c}{ MPE (dB) } \\
\cline { 2 - 5 } & Alternative & QMM & Alternative & QMM \\
\hline COST231-SU & 5.3327 & 6.0383 & -0.0053 & 0.0013 \\
COST231-UR & 5.3327 & 6.0383 & -0.0001 & 0.0023 \\
ECC33-L & 4.7423 & 5.5791 & -0.0034 & -0.0018 \\
ECC33-M & 4.7423 & 5.5791 & 0.0029 & -0.0031 \\
RGLI & 5.3327 & 6.0383 & -0.0009 & -0.0006 \\
ERICSSON & 5.3327 & 6.0383 & -0.0007 & -0.0042 \\
HATA & 5.3327 & 6.0383 & -0.0014 & 0.0028 \\
LEE & 5.3327 & 6.0383 & -0.0029 & 0.0045 \\
SUI & 5.3327 & 6.2032 & 0.0024 & -1.4206 \\
\hline
\end{tabular}

\subsection{Prediction Performances of Route B QMM Models}

Calibrated models for Route B are identified by (19)-(27), and the profiles of the pathloss predictions due to these models are displayed in Figures 4 and 5. The RMSE and MPE metrics of Table 3 represent evaluations of the prediction performances of these models, in comparison with pathloss measurements for the route.

Table 3. RMSE and MPE Metrics for Route B Models.

\begin{tabular}{lllll}
\hline \multirow{2}{*}{ Model/Metric } & \multicolumn{3}{l}{ RMSE (dB) } & MPE (dB) \\
\cline { 2 - 5 } & Basic & QMM & Basic & QMM \\
\hline COST231-SU & 9.9963 & 7.9652 & -0.3703 & 0.0051 \\
COST231-UR & 10.542 & 7.9653 & -3.3703 & 0.0013 \\
ECC33-L & 8.0903 & 7.6583 & -0.7309 & -0.0020 \\
ECC33-M & 20.494 & 7.6583 & -18.8447 & -0.0047 \\
RGLI & 27.701 & 7.9652 & 25.5832 & -0.0034 \\
ERICSSON & 65.267 & 7.9652 & -64.6496 & 0.0014 \\
HATA & 10.112 & 7.9653 & 1.5755 & 0.0008 \\
LEE & 15.744 & 7.9652 & 12.9271 & 0.0025 \\
SUI & 22.577 & 7.9652 & -18.9022 & -0.0024 \\
\hline
\end{tabular}

When compared with the corresponding metrics on Table 1, it is seen that RMSE values recorded in this case, as $7.6583 \mathrm{~dB}$ for ECC33 models and 7.9652 for all the other models (including the SUI-terrain A model), are poorer than those obtained for ROUTE A QMM models. And whereas the MAPE metrics for ROUTE B are also poorer than those recorded for ROUTE $\mathrm{A}$, the MPE metrics for the former route are better than those for the latter.

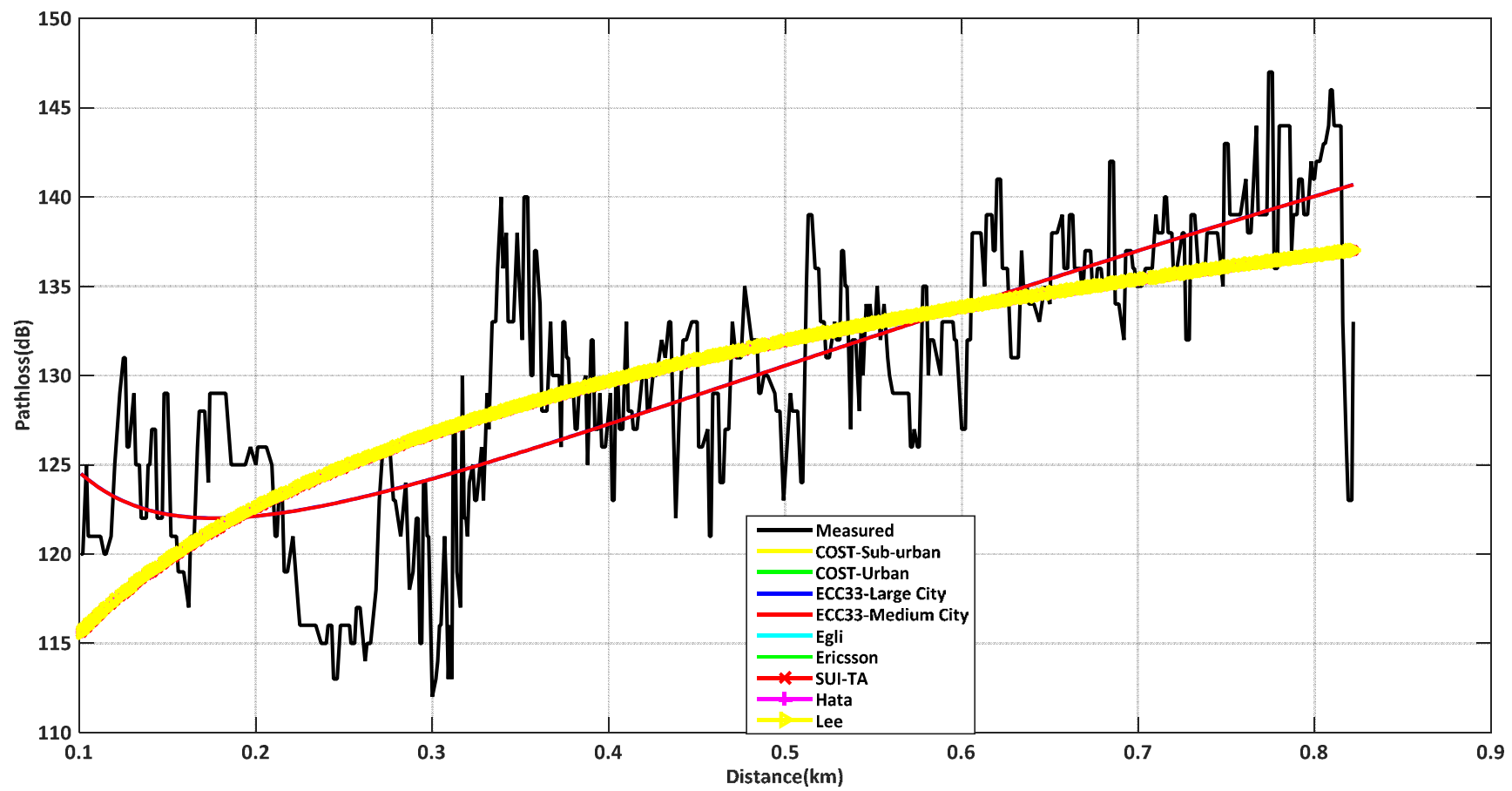

Figure 3. Comparison of predicted and measured pathloss for Route A Alternative QMM Models. 

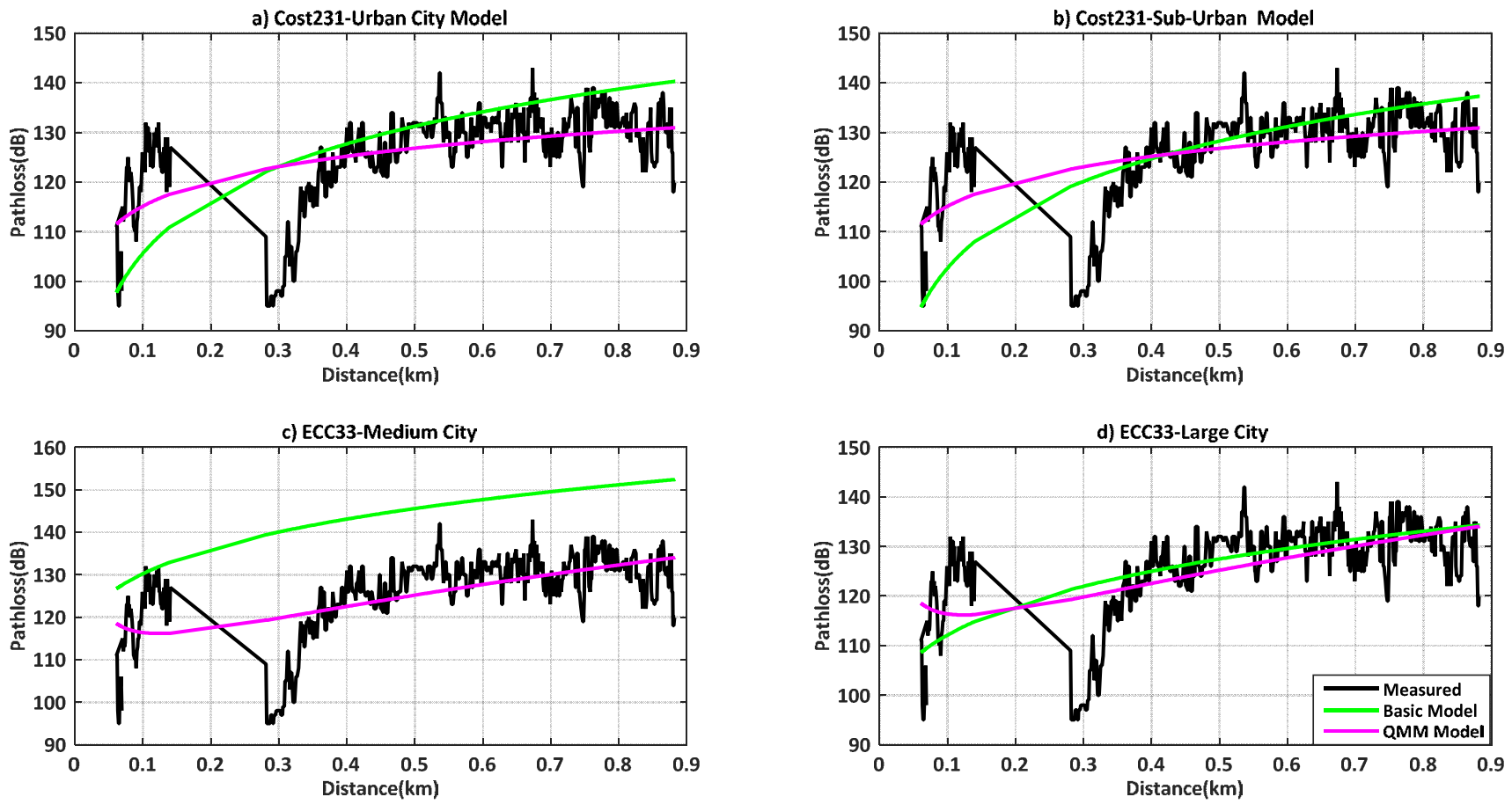

Figure 4. Comparison of predicted and measured pathloss for Route B QMM COST231 and ECC33 Models.
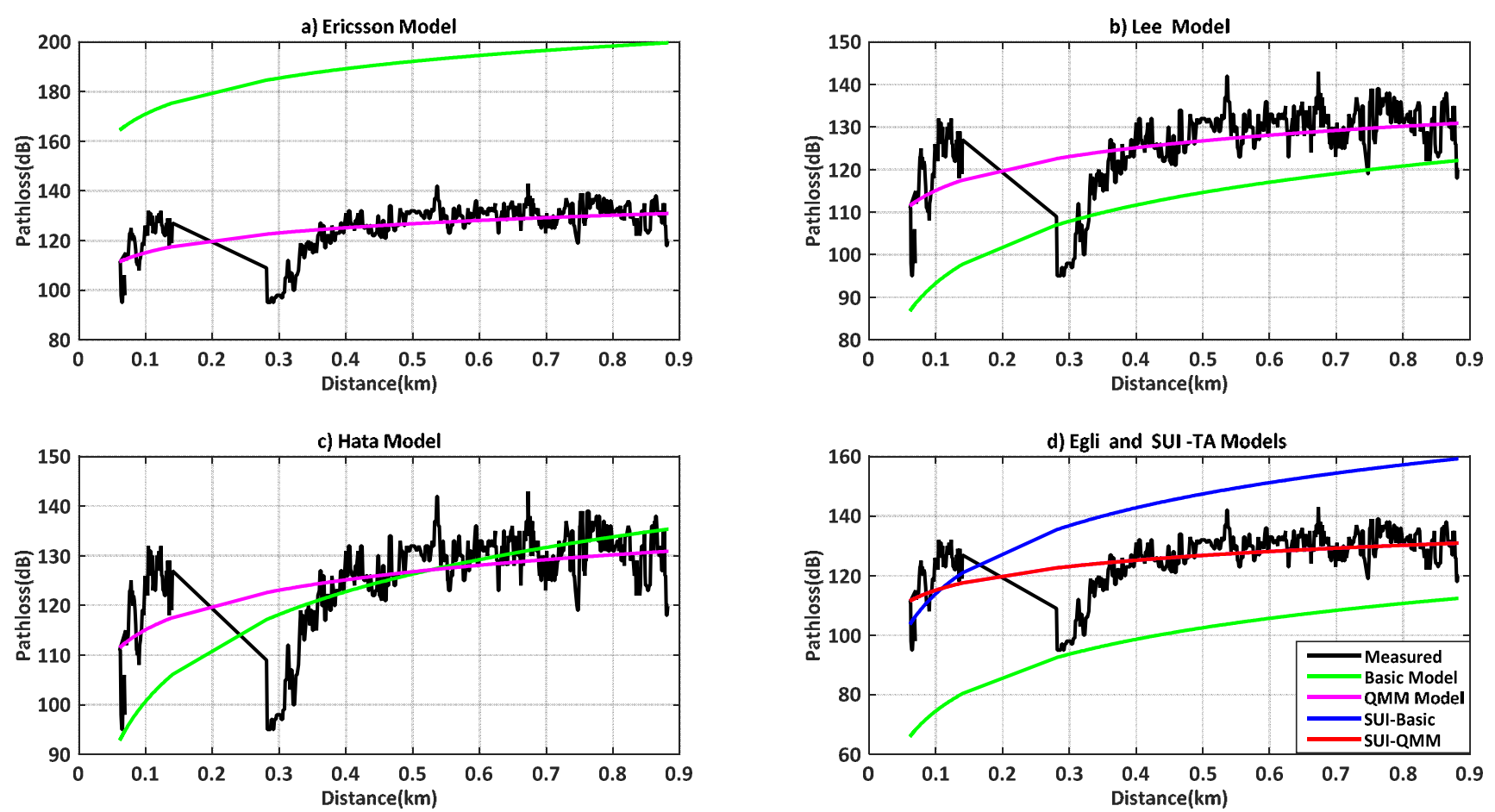

Figure 5. Comparison of predicted and measured pathloss for Route B QMM Egli, Ericsson, Hata, Leeand SUI Models. 


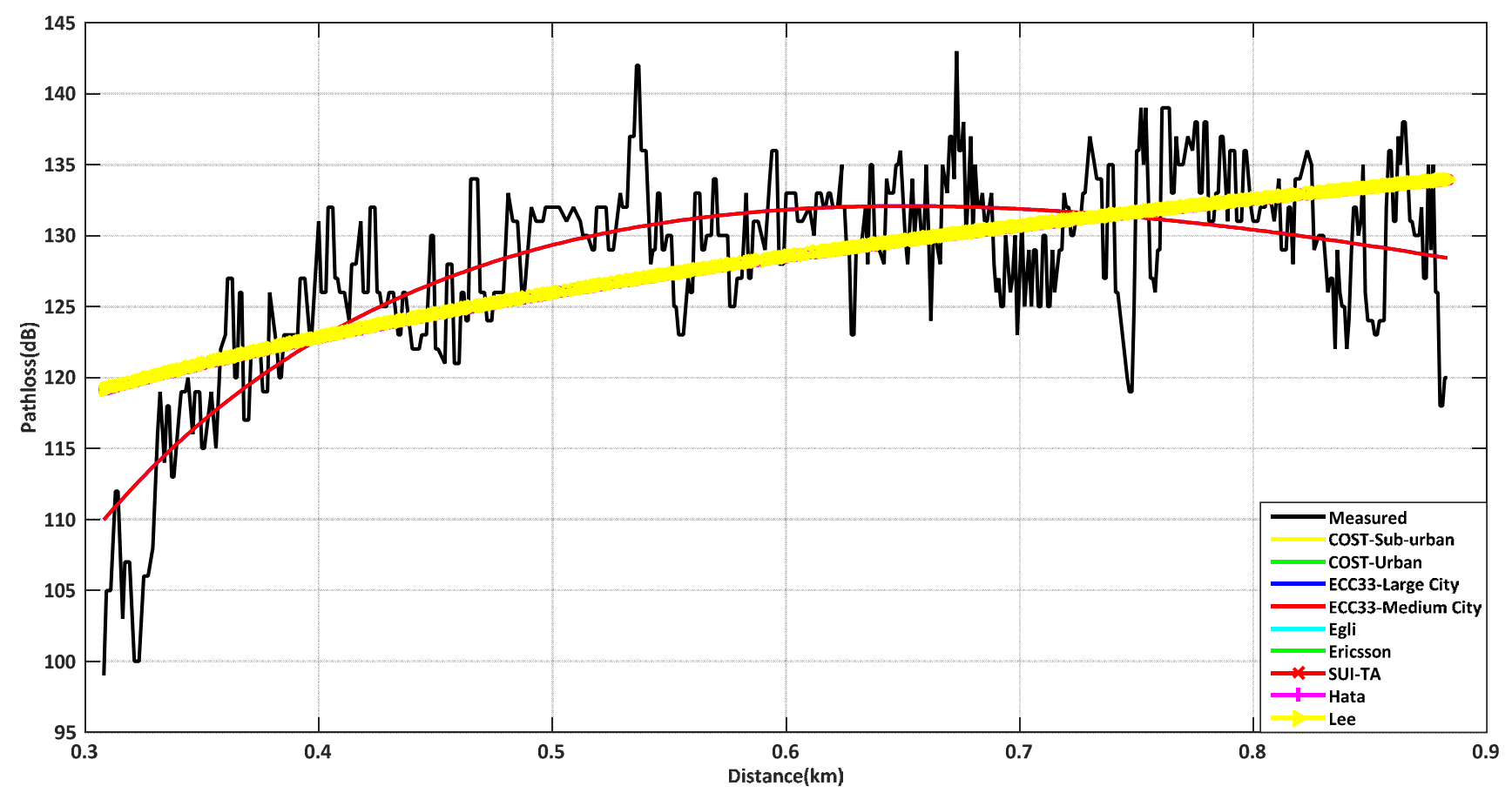

Figure 6. Comparison of predicted and measured pathloss for Route B Alternative QMM Models.

With the choice of measurement data utilized for calibration to obtain the alternative QMM models as defined by (46)-(54), the pathloss prediction profiles of Figure 6 emerged.

Table 4. RMSE and MPE Metrics for the Alternative Route B Models.

\begin{tabular}{lllll}
\hline \multirow{2}{*}{ Model/Metric } & RMSE (dB) & \multicolumn{3}{l}{ MPE (dB) } \\
\cline { 2 - 5 } & Alternative & QMM & Alternative & QMM \\
\hline COST231-SU & 5.3991 & 7.9652 & -0.0047 & -0.0119 \\
COST231-UR & 5.3991 & 7.9653 & -0.0044 & -0.0409 \\
ECC33-L & 4.3728 & 7.6583 & 0.0024 & -0.0020 \\
ECC33-M & 4.3728 & 7.6583 & 0.0021 & -0.0047 \\
RGLI & 5.3991 & 7.9652 & 0.0027 & -0.0034 \\
ERICSSON & 5.3991 & 7.9652 & -0.0007 & 0.0014 \\
HATA & 5.3991 & 7.9653 & -0.0409 & -0.0499 \\
LEE & 5.3991 & 7.9652 & -0.0064 & 0.0025 \\
SUI & 5.3991 & 7.9652 & 0.0032 & -0.0024 \\
\hline
\end{tabular}

RMSE and MPE metrics for these alternative models are presented in Table 4, from which it is readily observed that RMSE, for the ECC33 models, improved by about $43 \%$, and by about $33 \%$ for the other alternative models. It is apparent from these results, that the remarkable improvements recorded in this case owe in large part, to the exclusion, in the calibration process, of measurement data for $100 m \leq d \leq 308 m$, over which measured pathloss decreases virtually monotonically from $130 \mathrm{~dB}$ to $99 \mathrm{~dB}$.

\subsection{Prediction Performances of Route C QMM Models}

The QMM models for ROUTE $\mathrm{C}$ are those defined by (28)-(36), and whose pathloss prediction profiles are displayed in Figures 7 and 8.

The RMSE and MPE metrics for the calibrated models in the case of this route are displayed in Table 5 below.

Table 5. RMSE and MPE Metrics for Route C Models.

\begin{tabular}{lllll}
\hline \multirow{2}{*}{ Model/Metric } & \multicolumn{3}{l}{ RMSE $(\mathbf{d B})$} & MPE $(\mathbf{d B})$ \\
\cline { 2 - 5 } & Basic & QMM & Basic & QMM \\
\hline COST231-SU & 17.584 & 6.1944 & 8.5147 & -0.0015 \\
COST231-UR & 16.343 & 6.1944 & 5.5147 & 0.0053 \\
ECC33-L & 11.381 & 5.7926 & 6.3216 & 0.0008 \\
ECC33-M & 15.120 & 5.7926 & -11.7922 & 0.0060 \\
RGLI & 38.599 & 6.1944 & 34.7543 & -0.0059 \\
ERICSSON & 57.739 & 6.1944 & -56.3255 & 0.0006 \\
HATA & 18.604 & 6.1944 & 10.4606 & 0.0020 \\
LEE & 24.805 & 6.1944 & 21.2666 & -0.0009 \\
SUI & 22.277 & 6.1944 & -9.0217 & -0.0012 \\
\hline
\end{tabular}

The RMSE metrics for the calibrated, though comparable with those of corresponding ROUTE A models, are slightly poorer; but share the feature that they are identical for the two ECC33 models $(5.7926 \mathrm{~dB})$ on one hand, and the seven other models $(6.1944 \mathrm{~dB})$ on the other. As was the case with the other routes, excellent MPE metrics were recorded for all nine calibrated models, being in fact, virtually $0 \mathrm{~dB}$ for the calibrated ECC33 (large city), Ericsson and Lee models. It may also be remarked, that MAPE metrics for the calibrated models in this case are exactly the same only for the two ECC33 models, the differences between metrics for the other models may be considered small enough as to be insignificant. 

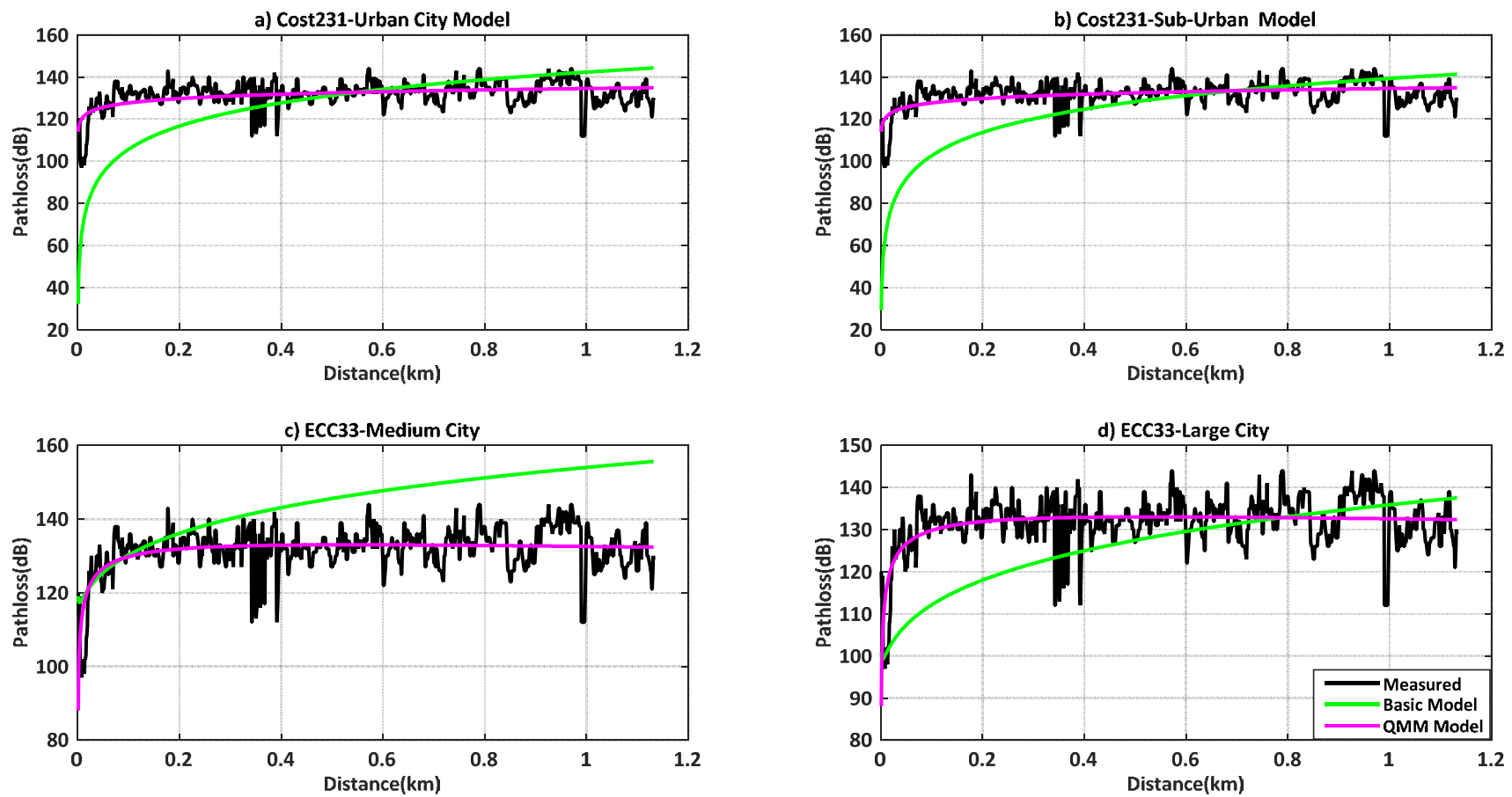

Figure 7. Comparison of predicted and measured pathloss for Route C QMM COST231 and ECC33 Models.
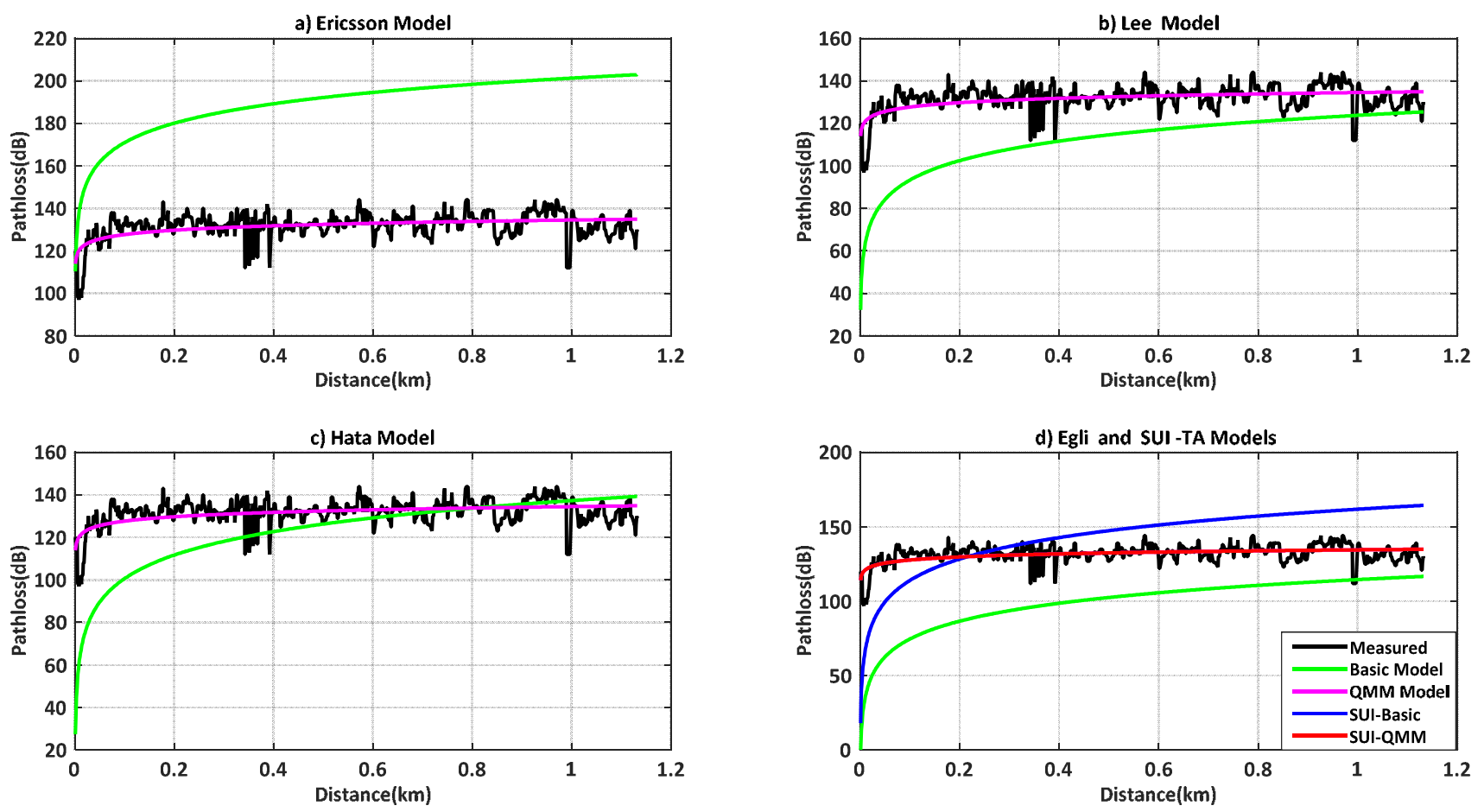

Figure 8. Comparison of predicted and measured pathloss for Route C QMM Egli, Ericsson, Hata, Lee and SUI Models.

Table 6. RMSE and MPE Metrics for the Alternative Route C Models.

\begin{tabular}{lllll}
\hline \multirow{2}{*}{ Model/Metric } & RMSE (dB) & \multicolumn{3}{c}{ MPE (dB) } \\
\cline { 2 - 5 } & Alternative & QMM & Alternative & QMM \\
\hline COST231-SU & 5.3610 & 6.1944 & -0.0003 & -0.0015 \\
COST231-UR & 5.3610 & 6.1944 & 0.0036 & 0.0053 \\
ECC33-L & 5.3603 & 5.7926 & 0.0011 & 0.0008 \\
ECC33-M & 5.3603 & 5.7926 & -0.0000 & 0.0060 \\
RGLI & 5.3610 & 6.1944 & -0.0013 & -0.0059 \\
ERICSSON & 5.3610 & 6.1944 & -0.0000 & 0.0006 \\
\hline
\end{tabular}

\begin{tabular}{lllll}
\hline \multirow{2}{*}{ Model/Metric } & RMSE (dB) & \multicolumn{3}{c}{ MPE (dB) } \\
\cline { 2 - 5 } & Alternative & QMM & Alternative & QMM \\
\hline HATA & 5.3610 & 6.1944 & -0.0011 & 0.0020 \\
LEE & 5.3610 & 6.1944 & 0.0050 & -0.0009 \\
SUI & 5.3610 & 6.1944 & -0.0019 & -0.0012 \\
\hline
\end{tabular}

Profiles of the pathloss predicted by the alternative models presented as (55)-(63) compared with the measurement data for the route are displayed in Figure 9. And the associated performance metrics (RMSE and MPE) are presented in 
Table 6.

It is readily verified that in this case, better improvements in RMSE $(13.5 \%)$ were recorded by the COST231, Egli, Ericsson, Hata, Lee, and SUI models than the two ECC33 models $(2.8 \%)$ unlike what obtained with the other two routes. Again, the MPE metrics for the models are excellent, and includes values of $0 \mathrm{~dB}$ for the ECC33 (small-medium city) and Ericsson models.

\subsection{Eigenvalues of the Model Calibration Matrix}

As was noted in [16], the real, symmetric model calibration matrices of the QMM calibration process are typically characterized by one predominant eigenvalue. In this section, the features of the eigenvalues associated with the nine basic pathloss prediction models are, for each of routes $\mathrm{A}, \mathrm{B}$, and $\mathrm{C}$, further explored, with reference to the influence of size of measurement data.

The distribution of the eigenvalues as obtained with the use of MATLAB's library function 'eig' for each of the basic models, and the three routes, are presented in what follows. It is evident from the profiles of Figure 10 that the model calibration matrices remain characterized by a single dominant eigenvalue, whose magnitude for route $\mathrm{C}$ is significantly greater than those for routes $\mathrm{A}$ and $\mathrm{B}$. The profiles also reveal that the magnitudes of the dominant eigenvalues for routes $\mathrm{A}$ and $\mathrm{B}$ are relatively close, and that for the alternative QMM models, route A's dominant eigenvalue has a larger magnitude than for route B's; the reverse being the case, when the full complement of measurement data points are utilized.
Indeed, the profiles of Figures 12 (for the Egli and Ericsson models) and 13 (for the Hata and Lee models) also display patterns similar to those described for the COST231 and ECC33 models, thus suggesting that it is legitimate to conclude that the pattern is characteristic of the model calibration matrices of QMM-based calibration of the basic pathloss models. The same remarks apply for the model calibration matrices of the ECC33 models, as can be seen from Figure 11.

It may be remarked here that a comparison of the metrics displayed in Tables 13, 14, and 15 of [14] with the corresponding metrics (Tables 1, 3, and 5) in this paper, very clearly show that the former utilized basic COST231 (sub-urban city) and ECC33 (medium-sized city) models for pathloss prediction. And according to the results of [4], the best performing (in terms of RMSE) basic models are COST231 $(9.8149 \mathrm{~dB})$ for Route A, $(9.9948 \mathrm{~dB})$ for Route B, and ECC33 (16.9762dB) for Route C. On the other hand, the results presented here reveal that the basic ECC33 (large city) is the best performing (7.5838dB-Route A; 8.0903dB-Route $\mathrm{B}$; and $11.381 \mathrm{~dB}$-Route C) across all three measurement routes The results also show that whereas the basic COST231 sub-urban city model had a better RMSE metric than the urban city model in the case of Route B, the latter's RMSE metrics were better than the former's for Route A (8.2309dB) and Route $\mathrm{C}(16.343 \mathrm{~dB})$. This suggests that when basic pathloss prediction models are being evaluated for any environment, it is best to investigative the performances of alternative (city size-based) models, where applicable.

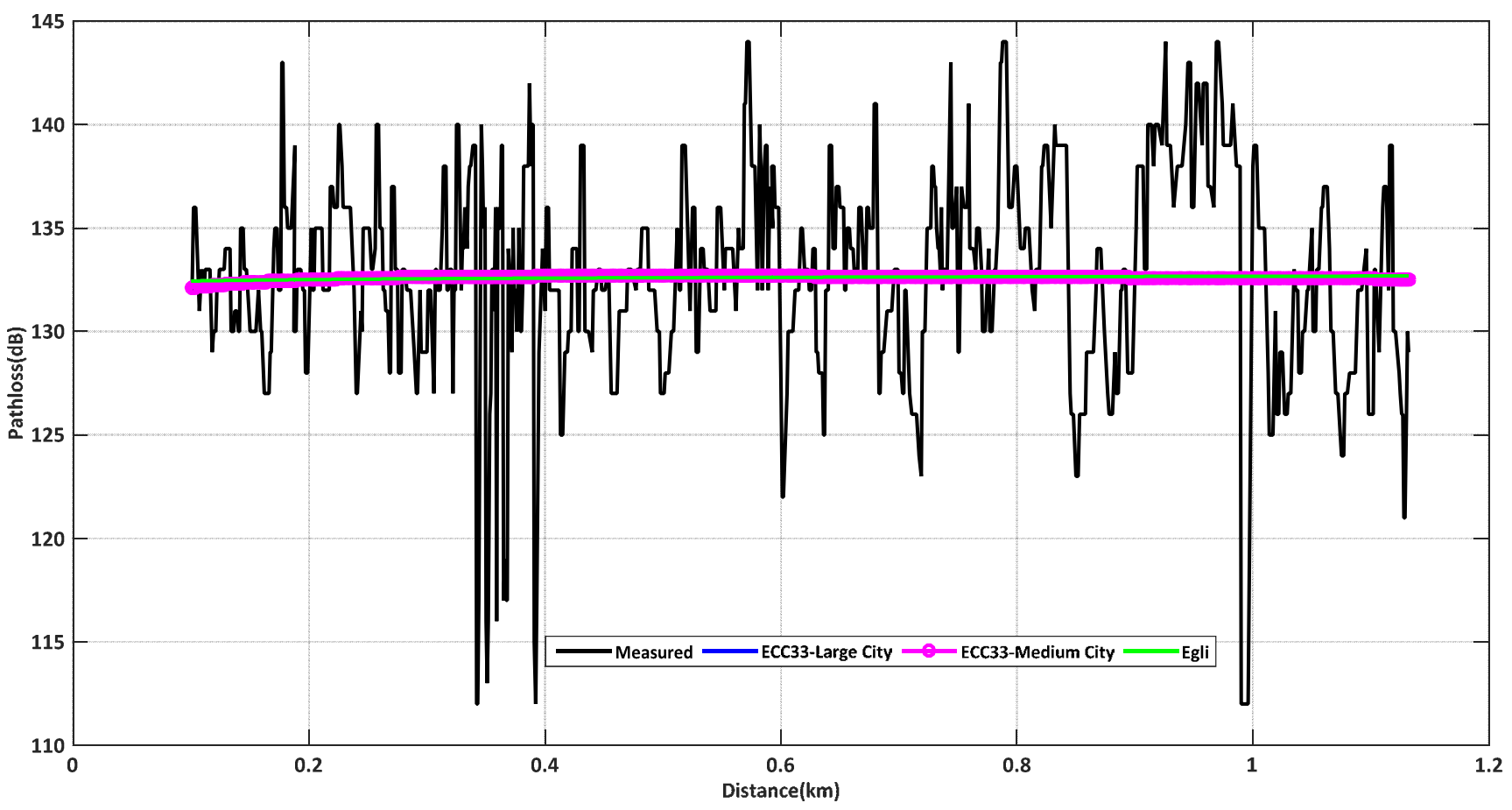

Figure 9. Comparison of predicted and measured pathloss for Route C Alternative QMM Models. 

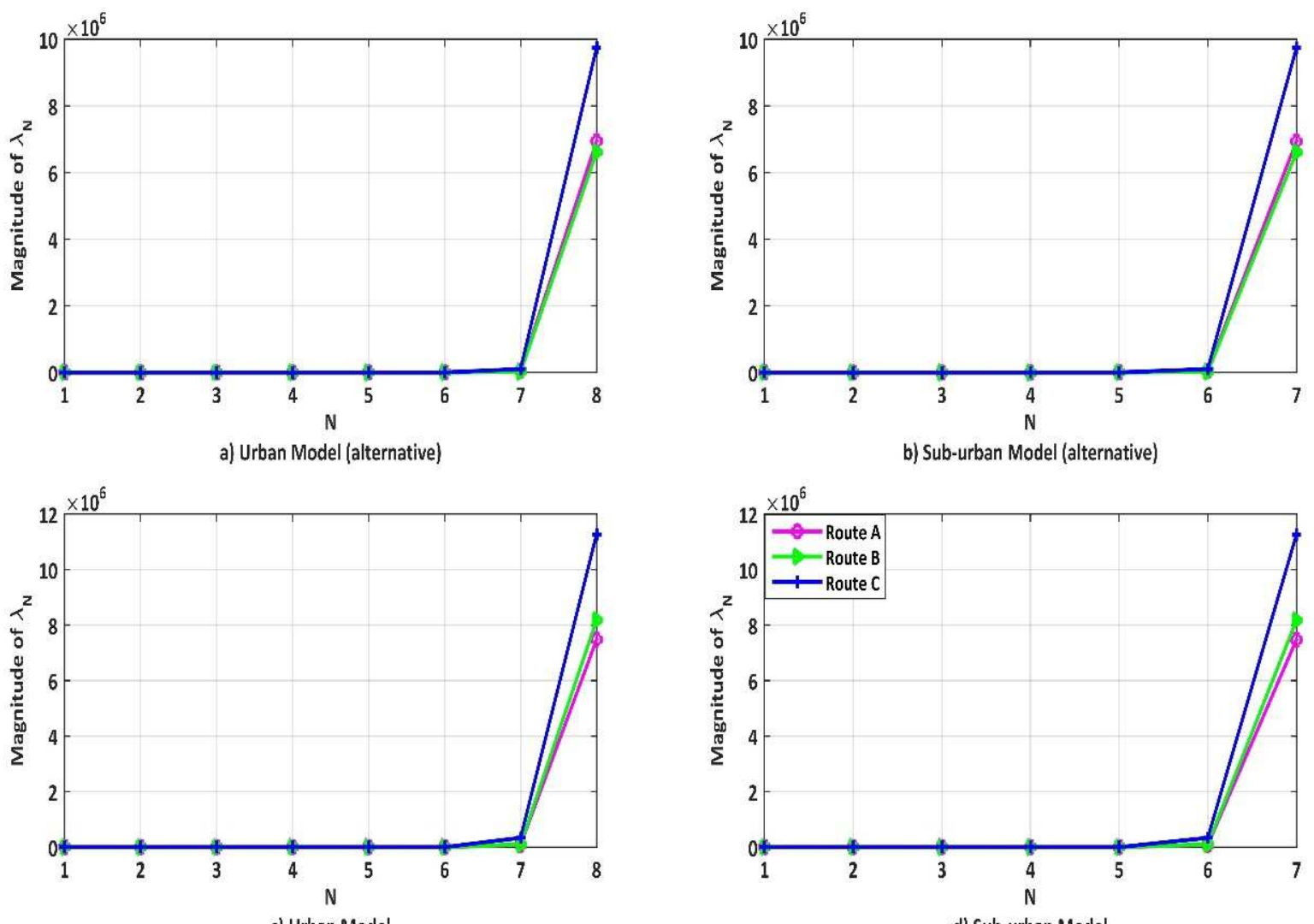

Figure 10. Eigen values of COST231 model calibration matrices.
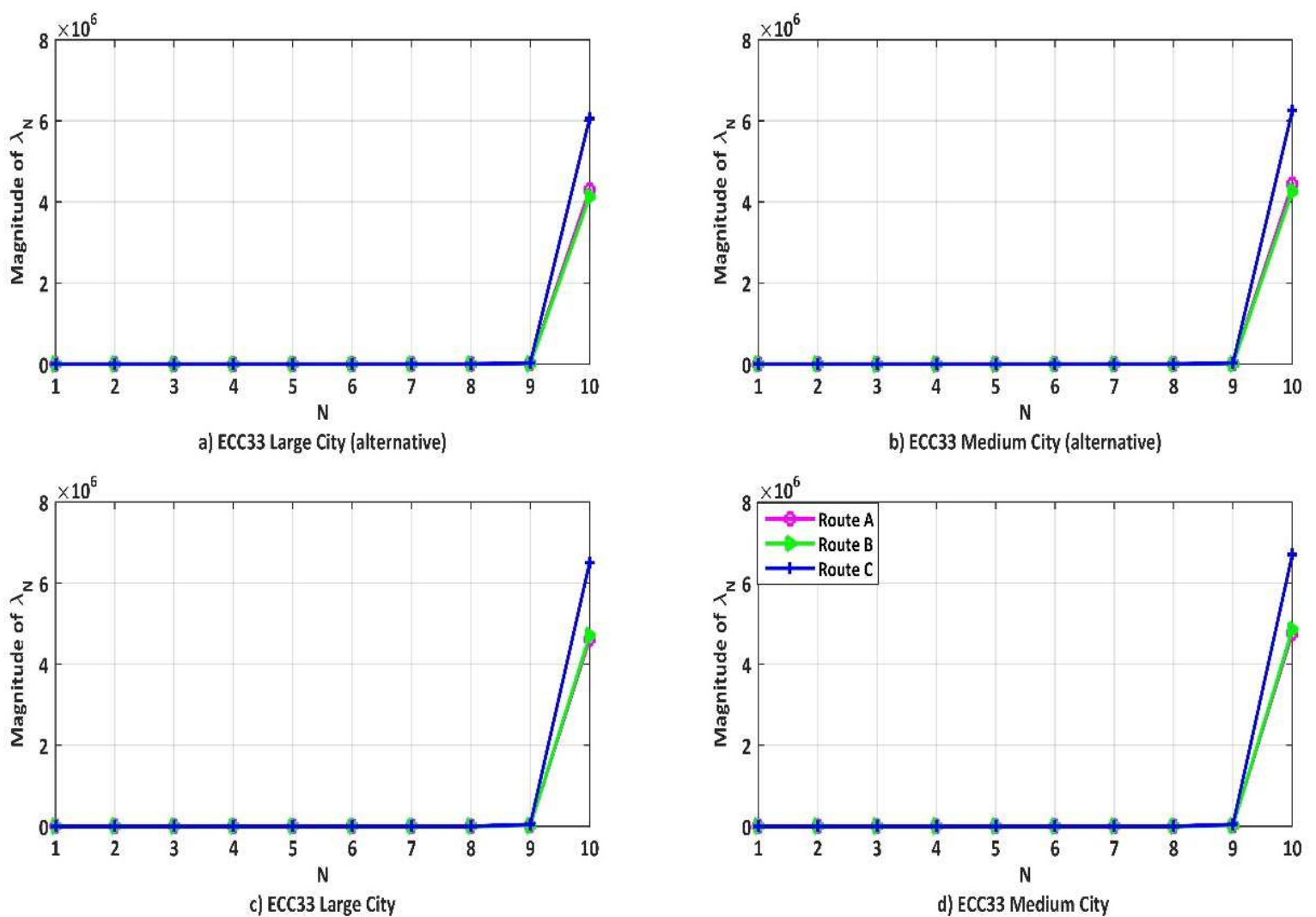

Figure 11. Eigenvalues of ECC33 model calibration matrices. 


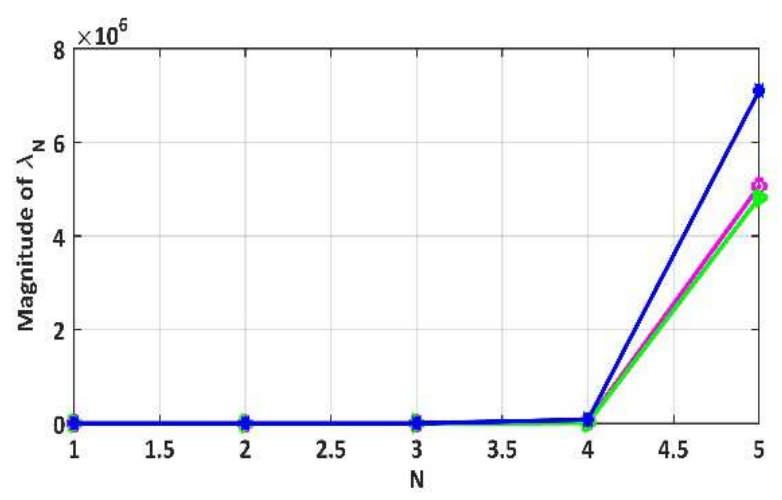

a) Egli Model (alternative)

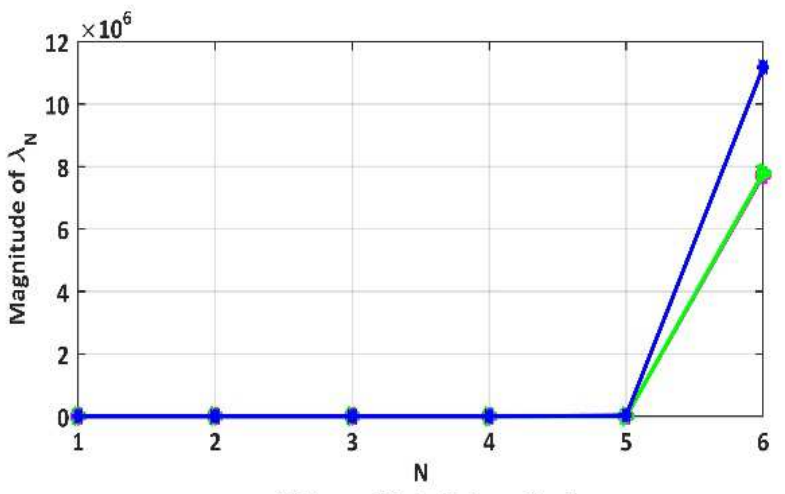

c) Ericsson Model (alternative)

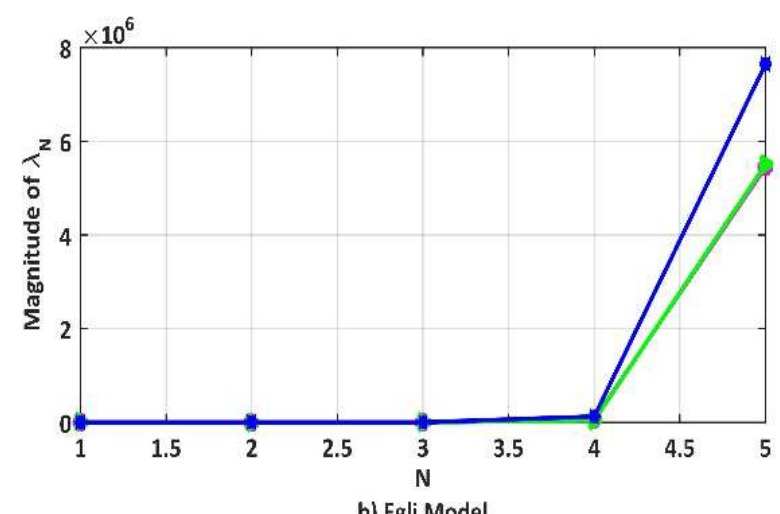

b) Egli Model

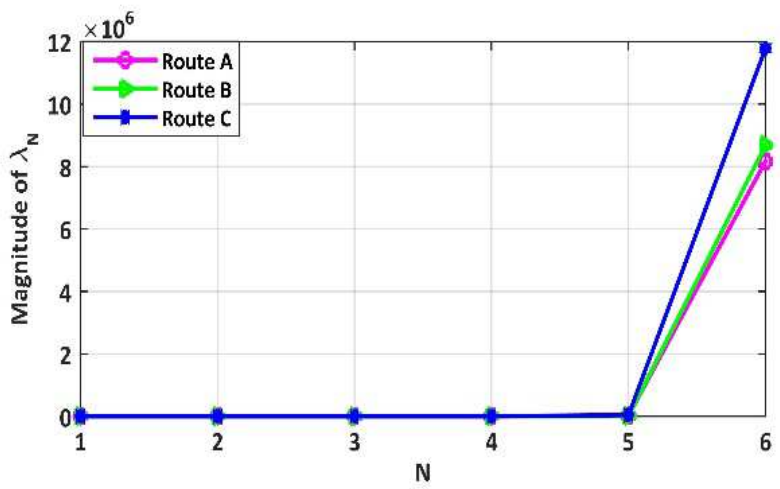

d) Ericsson Model

Figure 12. Eigenvalues of Egli and Ericsson model calibration matrices.

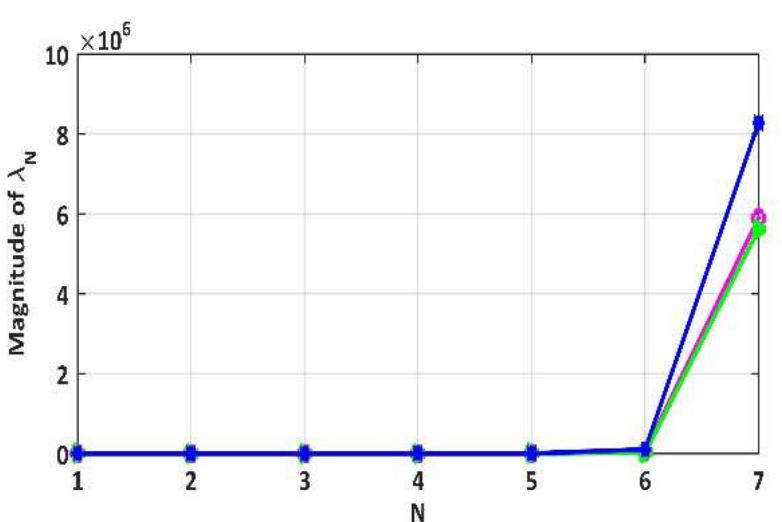

a) Hata Model (alternative)

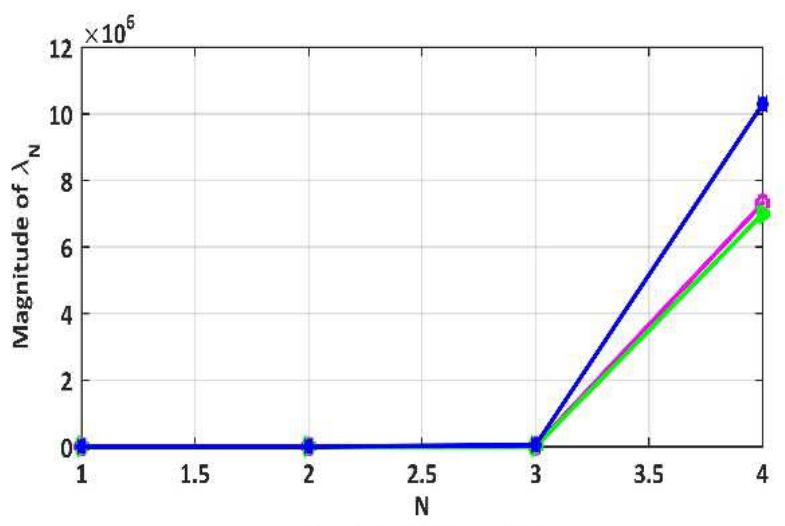

c) Lee Model (alternative)

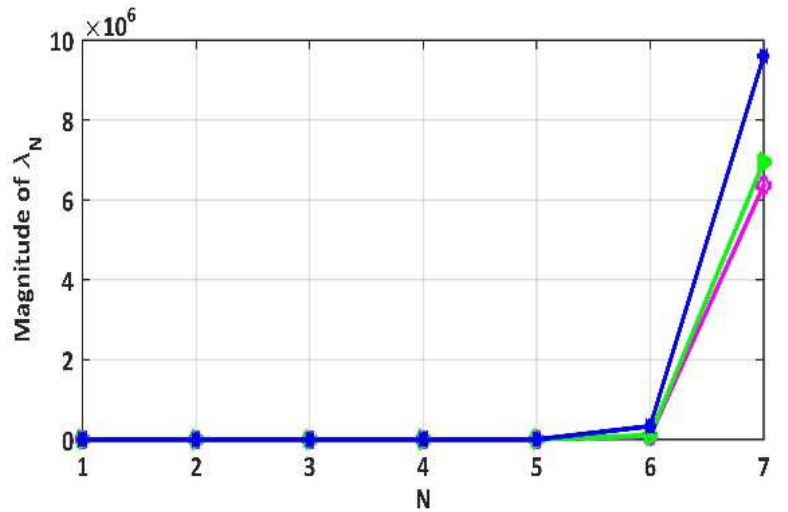

b) Hata Model

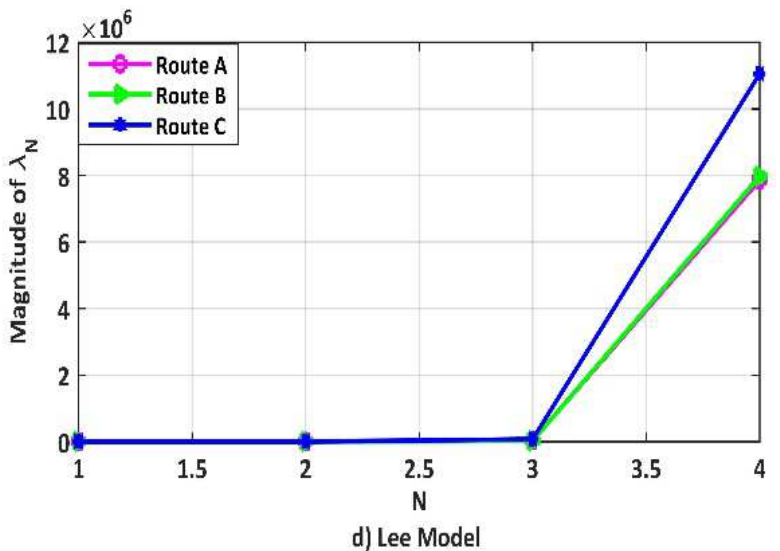

Figure 13. Eigenvalues of Hata and Lee model calibration matrices. 
In order to further explore the influence of data size on the magnitude of the dominant eigenvalues, the computed ratios of the largest dominant eigenvalues for route $\mathrm{C}$ to those of routes $\mathrm{A}$ and $\mathrm{B}$ are tabulated as shown in Table 7 , for the model calibration matrix of each basic model considered in this paper.

Table 7. Ratios of Dominant Eigenvalues: Route C: Routes A\&B.

\begin{tabular}{lllll}
\hline \multirow{2}{*}{ MODEL } & \multicolumn{2}{l}{ QMM } & \multicolumn{2}{l}{ Alternative $\mathbf{Q M M}$} \\
\cline { 2 - 5 } & $\mathbf{D E}_{\mathbf{C}} / \mathbf{D E}_{\mathbf{A}}$ & $\mathbf{D E}_{\mathbf{C}} / \mathbf{D E}_{\mathbf{B}}$ & $\mathbf{D E}_{\mathbf{C}} / \mathbf{D E} \mathbf{E}_{\mathbf{A}}$ & $\mathbf{D E}_{\mathbf{C}} / \mathbf{D E}_{\mathbf{B}}$ \\
\hline COST231-SU & 1.5074 & 1.3782 & 1.4045 & 1.4739 \\
COST231-UR & 1.5074 & 1.3782 & 1.4045 & 1.4739 \\
ECC33-L & 1.4091 & 1.3833 & 1.4107 & 1.4687 \\
ECC33-M & 1.4092 & 1.3831 & 1.4108 & 1.4686 \\
EGLI & 1.4020 & 1.3886 & 1.4039 & 1.4744 \\
ERICSSON & 1.4424 & 1.3571 & 1.4462 & 1.4355 \\
HATA & 1.5105 & 1.3898 & 1.4027 & 1.4755 \\
LEE & 1.4064 & 1.3855 & 1.4078 & 1.4716 \\
SUI & 1.4487 & 1.3511 & 1.4551 & 1.4257 \\
\hline
\end{tabular}

It is readily observed from the ratios in Table 7 that the ratios of magnitude of dominant eigenvalues for route $\mathrm{C}$ (denoted by $\mathrm{DE}_{\mathrm{C}}$ ) to those for both routes $\mathrm{A}$ and $\mathrm{B}$ (denoted by $\mathrm{DE}_{\mathrm{A}}$ and $\mathrm{DE}_{\mathrm{B}}$, respectively) are identical for the cases of the two COST231 models. This, put in other words implies, for example, that whereas the dominant eigenvalues $\left(\mathrm{DE}_{\mathrm{C}}\right.$, $\left.\mathrm{DE}_{\mathrm{A}}\right)$ are different for the model calibration matrices of the COST231 (urban city and sub-urban city) models, the ratios $\left(\frac{D E_{C}}{D E_{A}}\right)_{C O S T-U R B}$ and $\left(\frac{D E_{C}}{D E_{A}}\right)_{C O S T-S U B}$ give exactly the same numerical value.

Table 8. Ratios of Dominant Eigenvalues: $Q M M: Q M M_{\text {alt: }} \&(A: B)_{\text {alternative. }}$

\begin{tabular}{lllll}
\hline \multirow{2}{*}{ MODEL } & \multicolumn{3}{l}{ DE $_{\text {QMM }} / \mathbf{D E}_{\mathbf{Q M M}-A L T}$} & \multirow{2}{*}{ DE $_{\mathbf{A}} / \mathbf{D E}_{\mathbf{B}}$} \\
\cline { 2 - 4 } & ROUTE-A & ROUTE-B & ROUTE-C & \\
\hline COST231-SU & 1.0773 & 1.2366 & 1.1563 & 1.0495 \\
COST231-UR & 1.0773 & 1.2366 & 1.1563 & 1.0494 \\
ECC33-L & 1.0737 & 1.1387 & 1.0725 & 1.0411 \\
ECC33-M & 1.0736 & 1.1387 & 1.0724 & 1.0410 \\
EGLI & 1.0776 & 1.1426 & 1.0762 & 1.0502 \\
ERICSSON & 1.0572 & 1.1153 & 1.0544 & 0.9926 \\
HATA & 1.1779 & 1.2390 & 1.1596 & 1.0519 \\
LEE & 1.0751 & 1.1408 & 1.0704 & 1.0453 \\
SUI & 1.0547 & 1.1081 & 1.0501 & 0.9798 \\
\hline
\end{tabular}

It should be remarked that the entries in Table 7 do not reflect the relative magnitudes of the dominant eigenvalues. For the computational results reveal in the case of route $\mathrm{C}$, for example, the descending order of magnitudes is Ericsson, Lee, COST231 (urban city), COST231 (suburban city), Hata, Egli, EC33 (small-medium sized city), ECC33 (large city), and SUI models, when relevant data for the alternative calibration is utilized; and when the full complement of measurement data is utilized, the order recorded is Ericsson, COST231 (Urban and suburban, in that order), Lee, Hata, Egli, ECC33 (medium city and large city, in that order), and SUI models. Similar items of information for routes A and B are summarized by Table 8 , which displays the ratios of dominant eigenvalues of the alternative QMM models.

\section{Conclusions}

This paper, using measurement data made freely available in the literature, has examined certain properties of the recently introduced Quasi-Moment-Method (QMM) for the calibration of basic radiowave propagation pathloss models. In particular, the influence of large-sized field measurement data (in excess of 400, in this case) on the prediction performances of the calibrated models was examined.

And a number of interesting features were revealed, by the computational results, for the model calibration tool. First, for the basic COST231, Egli, Ericsson, Hata, Lee, and SUI models (which, for given frequency operation, transmitter antenna and receiver antenna heights, have the generic form $\left.\alpha_{m}+\beta_{m} \log _{10} d\right)$ results of QMM calibration converged to the same RMSE solution as can be seen from Tables 1-6. This same observation is shown to be true by the results of Tables 1-6 for the ECC33 models, whose corresponding generic form is $a_{m}+b_{m} \log _{10} d+c_{m}\left(\log _{10} d\right)^{2}$. It is important to note that the constants $\left(\alpha_{m}, \beta_{m}, a_{m}, b_{m}, c_{m}\right)$ appearing in the forgoing remarks, vary from basic model to basic model. It is also worth pointing out that whereas the Mean Prediction Error (MPE) values differ for the two sets of calibrated models, the Mean Absolute Prediction Errors (MAPE) converged for the two sets, as did the RMSE metrics.

Second, the results suggested that when model calibration is restricted to regions farther (about $100 \mathrm{~m}$ ) away from the transmitter than provided for in the original measurement data, significant improvements in the Root Mean Square Error (RMSE) metrics of the models can be expected. As examples, prediction RMSE improved for the pair of ECC33 models, from $5.5797 \mathrm{~dB}$ to $47423 \mathrm{~dB}(15 \%)$, in the case of Route A, $7.6583 \mathrm{~dB}$ to $4.3728 \mathrm{~dB}(43 \%)$ for Route $\mathrm{B}$, and from $5.7926 \mathrm{~dB}$ to $5.3603 \mathrm{~dB}(7.5 \%)$, for Route $\mathrm{C}$. The same trend was recorded for the group of other models, with RMSE improvements ranging from $9 \%$ for Route $\mathrm{C}$, through $12 \%$ for Route A, to $37 \%$ for Route B. Third the results confirmed the conclusions reported by earlier investigations, that in terms of RMSE, the ECC33 models respond best to QMM calibration; and that QMM calibrated models in general, provide excellent Mean Prediction Error (MPE) metrics, approaching $0 \mathrm{~dB}$ in quite a few cases. Finally, and from the results due to the alternative QMM models for 'ROUTE B, it may be concluded that although MPE metrics are, compared to RMSE metrics, only slightly affected by the presence of a significant number of outliers in measurement data, RMSE metrics, on the other hand, improve tremendously, when the outliers are excluded from the calibration process.

The paper also described properties of the eigenvalues of the model calibration matrices associated with the basic models, as a preliminary step towards the possible formulation of an eigenvalue problem for the empirical pathloss development process. Each model calibration matrix, the results reveal, is characterized by one dominant eigenvalue, whose properties of apparent interest to future investigations 
are described in some details, in section 3.4 of the paper.

\section{References}

[1] Durgin, G.,. Rappaport, T. S., \&. Xu, H, (1998). 5.85-GHz radio path loss and penetration loss measurements in and around homes and trees," in IEEE Communications Letters, vol 2, no. 3, pp. 70-72, doi: http://10.1109/4234.662630.

[2] Cheng, W., Liu, T., Hsu, M,. Tsai, Z., and. Sheen, W., (2015). $15 \mathrm{GHz}$ propagation channel measurement at a university campus for the 5G spectrum, 2015 Asia-Pacific Microwave

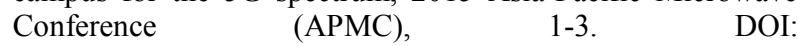
http://10.1109/APMC.2015.7413114.

[3] Aborahama, M., Zakaria, A., Ismail, M. H., El-Bardicy, M., El- Tarhuni, M. \& Hatahet, Y (2020) Large-scale channel characterization at $28 \mathrm{GHz}$ on a university campus in the United Arab Emirates, Telecommunication Systems 74: 185199 DOI: https://doi.org/10.1007/s11235-019-00649-6.

[4] Nwawelu, U. N., Nzeako, A. N., \&Ahaneku, M. A.(2012). The Limitations of Campus Wireless Networks: A Case Study of University of Nigeria, Nsukka [Lionet] International Journal of Networks and Communications 2012, 2 (5): Pp. 112-122 DOI: http://10.5923/j.ijnc.20120205.04.

[5] Fraga-Lamas, P., Celaya-Echarri, M., Lopez-Iturri, P., Castedo, L., Azpilicueta, L. Aguirre, E. Suárez-Albela, M., Falcone, F.\& Fernández-Caramés, T. M. (2019) Design and Experimental Validation of a LoRaWAN Fog Computing Based Architecture for IoT Enabled Smart Campus Applications Sensors 2019, 19, 3287; DOI: http://10.3390/s19153287.

[6] Han, S. Y., Abu-Ghazaleh, N. B., \&. Lee, D. (2016) Efficient and Consistent Path Loss Model for Mobile Network Simulation," in IEEE/ACM Transactions on Networking, vol. 24, no. 3, 1774-1786, doi: http://10.1109/TNET.2015.2431852 96.

[7] Ogunjide, S. B., Ohize, H. O. Usman, A. U., Abiodun, E., Adrgboye, M. A.,\& Salami, H. T.(2020). Suitable Propagation Models for $2.4 \mathrm{GHz}$ Wireless Networks: Case Study of Gidan Kwano Campus, FUT MINNA, ABUAD Journal of Engineering Research and Development (AJERD), Vol. 3 (1). 156-165.

[8] Ramos G, Vargas C, Mello L, Pereira P, Vieira R, Gonçalves \& S, Rego, C. (2020): Measurement and Prediction of Short-Range Path Loss between 27 and $40 \mathrm{GHz}$ in University Campus Scenarios. Research Square 1-14 DOI: 10.21203/rs.3.rs-70739/v1.

[9] Olajide, O. Y. and Samson, Y. M. (2020). Channel Path-Loss Measurement and Modeling in Wireless Data Network (IEEE 802.11n) Using Artificial Neural Network EJECE, European Journal of Electrical and Computer Engineering Vol. 4, No. 1. 1-7 DOI: http://dx.doi.org/10.24018/ejece.2020.4.1.157.

[10] Femi-Jemilohun, O. J. \& Walker S. D, (2014). Path loss prediction models for Corridor propagation at $24 \mathrm{GHz}$ Transactions on Networks and Communications Vol 2 (4). 84-94. DOI: $\quad 10.14738 /$ tnc.24.361 http://dx.doi.org/10.14738/tnc.24.361.

[11] Ogbeide, K. O.,\& Aikhoje, P. T (2017). Investigation of GSM signal propagation and models verification in the University of Benin, Ugbowo Campus Journal of Nigerian Association of Mathematical Physics, Vol v0, 2017. Pp. 437-332.

[12] Oyetunji, S. A. (2013), Determination of Propagation Path Loss and Contour Map for FUTA FM Radio Federal University of Technology, Akure IOSR Journal of Electronics and Communication Engineering (IOSR-JECE) Volume 6, Issue 3 (May. - Jun. 2013), 4-9.

[13] De Luca, D., Fiano, F., Mazzenga, F., Monti, C., Ridolfi, S. \&. Vallone, F. (2007). Outdoor Path Loss Models for IEEE 802.16 in Suburban and Campus-Like Environments, 2007 IEEE International Conference on Communications, 2007, pp. 4902-4906, DOI: http://10.1109/ICC.2007.809.

[14] Popoola, S. I, Atayero, A. A.,\& Popoola, O. A (2018). Comparative assessment of data obtained using empirical models for path loss predictions in a university campus environment Data in BriefVolume 18, June 2018,. 380-393 https://doi.org/10.1016/j.dib.2018.03.040.

[15] Popoola, S. I, Atayero, A. A, Arausi, O. D., \& Matthews, V. O. (2018). Path loss dataset for modeling radio wave propagation in smart campus environment; Data in Brief Volume 17, April 2018, Pp. 1062-1073 https://doi.org/10.1016/j.dib.2018.02.026.

[16] Adekola, S. A., Ayorinde, A. A., Okewole, F. O. \& Ike Mowete (2021) A Quasi-Moment-Method empirical modelling for pathloss prediction, International Journal of Electronics Letters, DOI: 10.1080/21681724.2021.1908607.

[17] Ayorinde, A. A, Muhammed, H. A., \&Mowete, Ike. (2021). On the Response of Basic Walfisch-Ikegami and Walfisch-Bertoni Models to QMM Calibration. International Journal of Computer Applications. 174. Pp. 53-64. http://10.5120/ijca2021921100.

[18] Mowete, Ike, Adelabu, M. A., Ayorinde, A. A., Muhammed, H. A., \& Okewole, F. O (2020) A Quasi-Moment-Method-Based Calibration of Basic Pathloss Models ELEKTRIKA, Journal of Electrical Engineering, VOL. 19, NO. 3, 2020, 35-48 http://10.11113/ELEKTRIKA.V19N3.232. 